\title{
Analytical Expressions for High-Frequency VLSI Interconnect Impedance Extraction in the Presence of a Multilayer Conductive Substrate
}

\author{
Navin Srivastava, Student Member, IEEE, Roberto Suaya, Senior Member, IEEE, and \\ Kaustav Banerjee, Senior Member, IEEE
}

\begin{abstract}
We propose an efficient method to accurately compute the frequency-dependent impedance of VLSI interconnects in the presence of multilayer conductive substrates. The resulting accuracy (errors less than 3\%) and CPU time reduction (more than an order of magnitude) emerge from three different ingredients: a 2-D Green's function approach with the correct quasi-static limit, a modified discrete complex images approximation to the Green's function, and a continuous dipole expansion to evaluate the magnetic vector potential at the short distances that are relevant to VLSI interconnects. This approach permits the evaluation of the self-impedance and mutual-impedance of multiconductor current loops, including substrate effects, in terms of easily computable analytical expressions that involve their relative separations and the electromagnetic parameters of the multilayer substrate.
\end{abstract}

Index Terms-Green's function, high frequency, impedance, magnetic dipole, parasitic extraction, substrate, VLSI interconnect.

\section{INTRODUCTION}

A S VERY large scale integration (VLSI) technology continues to scale, the number of wires in an integrated circuit, as well as their impact on circuit delay, noise, and power dissipation, increases rapidly. Hence, parasitic extraction techniques that are computationally efficient as well as reasonably accurate are indispensable. However, interconnect parasitic extraction presents a challenging task owing to the sheer size of the problem both in terms of computation time and required memory. The complexity of the problem is further compounded as lithography scaling enables faster transistors, driving maximum signal propagation frequencies on interconnects into the 20 - to $100-\mathrm{GHz}$ range [1]. In this enlarged frequency regime, it is imperative to analyze the effect

Manuscript received May 16, 2008; revised October 4, 2008 and December 19, 2008. Current version published June 17, 2009. This work was supported by Mentor Graphics Corporation under the University of California-MICRO program (Grant 06-243, Grant 07-095, and Grant 08-116). This paper was recommended by Associate Editor L. M. Silveira.

N. Srivastava was with the Department of Electrical and Computer Engineering, University of California, Santa Barbara, CA 93106 USA. He is now with the Calibre Division, Mentor Graphics Corporation, Wilsonville, OR 97070 USA (e-mail: navins@ece.ucsb.edu).

R. Suaya is with the Calibre Division, Mentor Graphics Corporation, 38334 St. Ismier, France (e-mail: roberto_suaya@mentor.com).

$\mathrm{K}$. Banerjee is with the Department of Electrical and Computer Engineering, University of California, Santa Barbara, CA 93106 USA (e-mail: kaustav@ece.ucsb.edu).

Color versions of one or more of the figures in this paper are available online at http://ieeexplore.ieee.org.

Digital Object Identifier 10.1109/TCAD.2009.2017432

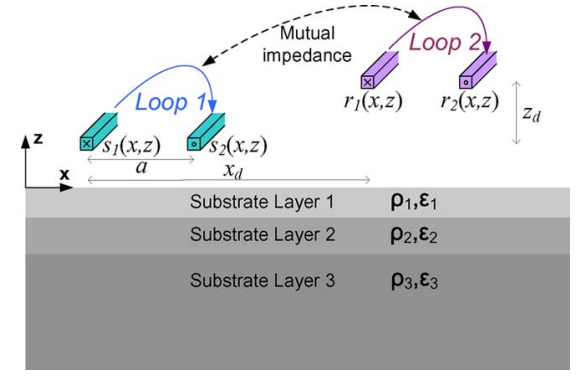

Fig. 1. Schematic of a general interconnect configuration with magnetic interaction between two conductor loops: Loop1 (source of magnetic field) and Loop2 (victim). Signal wires ( $s_{1}$ and $\left.r_{1}\right)$ are identified by crosses and ground wires (return paths, $s_{2}$ and $r_{2}$ ) by dots.

on interconnect circuit parameters arising from the presence of complex substrate structures underneath the interconnect layers. The underlying physics is not difficult to visualize. Transient currents in interconnects are the sources of timevarying magnetic fields, which, in turn, induce currents in other interconnects as well as eddy currents in the lossy substrate. The presence of these eddy currents modifies the impedance matrix $[Z]$ of the interconnects.

At high frequencies, the effect of a low-resistivity substrate on interconnect impedance $(Z=R+j \omega L)$ is a matter of significant concern [2]. Often, a very high resistivity $(\sim 1000 \Omega \cdot \mathrm{cm})$ substrate is used (underlying a low-resistivity surface layer for active devices) in radio-frequency or mixed-signal ICs [3] to substantially decrease the importance of substrate eddy currents. However, low-resistivity substrates continue to be used for latch-up avoidance [2]. Hence, to efficiently and accurately compute the impact of the multilayer substrate on interconnect impedance, we are compelled to introduce a parasitic extraction methodology that incorporates this effect.

We investigate the problem of frequency-dependent selfimpedance and mutual-impedance extraction for interacting conductor loops in a VLSI circuit in the presence of a multilayer conductive substrate, as schematically shown in Fig. 1. Prevalent interconnect extraction tools are too expensive in terms of computation time and/or memory to handle this problem. For example, in the industry standard tool FastHenry [4], which uses the free-space Green's function, the substrate must be specified as an explicit conductive layer(s) demanding several thousands of filaments at high frequencies. The resulting linear system is rapidly overwhelmed by the size requirements related to the partitioning of the substrate even for single-layer 


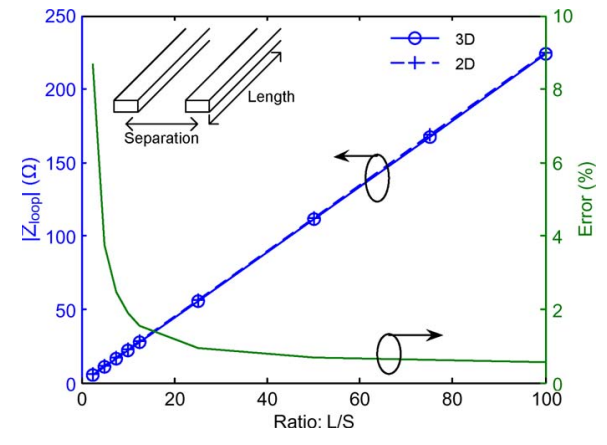

Fig. 2. (Left $y$-axis) Comparison of 2-D loop-impedance computation with 3-D computation as a function of the ratio of conductor lengths to transverse separation $(L / S)$ in the presence of a three-layer substrate at $100 \mathrm{GHz}$. (Right $y$-axis) Error (\%) due to the 2-D approximation.

substrate media. This constitutes orders-of-magnitude overhead in computation time and memory requirement even for the simplest interconnect configurations.

A natural alternative is to construct a modified Green's function, wherein the substrate's presence is implicitly reflected through the boundary conditions. No explicit reference to the substrate remains while computing the fields above it-only the interconnects need to be considered explicitly-thereby enormously reducing the dimensionality of the linear system. However, these implicit Green's functions are themselves more expensive to compute.

In the partial element equivalent circuit (PEEC) quasi-static formalism [5] in two dimensions, several authors have studied implicit Green's functions with a single open current-a magnetic monopole - as the current source above the substrate [6], [7]. These works utilize a complex image approximation to the Green's function, which works well for antenna applications with large separations between the source current and the observation point [8], leading to analytical expressions. For shorter separations, as relevant to VLSI applications, these approximations become inaccurate, as discussed in Section II. Another approach presented in [9] includes more complex approximations that are computationally expensive. The different approaches to compute the implicit Green's function, as well as their shortcomings, are briefly reviewed in Section II. We are interested in an alternative that is accurate yet computationally inexpensive.

For VLSI interconnect parasitic extraction, we rely on a loop-impedance formalism [10], leading to the correct physical behavior of closed on-chip currents. In this approach, the longdistance behavior of impedance parameters falls off as a power law, instead of the unphysical logarithmic behavior in the PEEC [5]. Hence, in the loop formalism, the separations between conductor loops with appreciable mutual impedances are upperbounded by a few tens of micrometers [11] for current and future technology nodes, and larger separations can be neglected.

We are interested in computing the high-frequency impedance matrix for interconnects that are sensitive to inductance effects. The length of such interconnects is greater than $100 \mu \mathrm{m}$ [1], which is much larger than the transverse separations where mutual impedance is appreciable. Hence, a 2-D treatment of these interconnects is valid. Fig. 2 shows that the error in loop impedance due to the 2-D approximation is small when the conductor lengths exceed 20 times the transverse separations between them.
For the impedance extraction of Manhattan interconnects (see Fig. 18), the collection of a signal wire and its return paths is partitioned along their length to form bundles [10], such that all wires in a bundle have the same length. As discussed above, the common length of the conductors in a bundle must be at least 20 times larger than the separations between them to permit the 2-D approximation. Timing-critical global interconnects that are designed to keep inductive effects under control often satisfy this constraint. In this case, the self-impedance of each bundle and the mutual impedance between adjacent parallel bundles can be computed using a 2-D approach. Bundles that are perpendicular to each other have no mutual interaction, whereas the mutual impedance between bundles that are far apart can be neglected. The approach described in this paper can, thus, be applied to Manhattan VLSI interconnects ${ }^{1}$ subject to the constraint that each segment of the Manhattan wire is long and has nearby return paths.

To describe the current-loop interactions, in Section III, we compute the Green's function for a magnetic dipole in the presence of a multilayer substrate. This Green's function describes the 2-D problem in the magneto-quasi-static (MQS) approximation [12], which allows us to neglect displacement currents when the length scales of interest are smaller than the wavelength of light in the medium. Up to the maximum frequency of interest for VLSI technology (100 GHz), a quasi-static computation of the magnetic vector potential is justified since the minimum wavelength $(>1.5 \mathrm{~mm})$ is larger than the relevant transverse dimensions of the interconnect geometry. However, the skin depth of the substrate layers is often of the same order as the wavelength. Hence, the quasi-static assumption used in this paper is later validated by comparing with a full-wave field solver (see Fig. 15) after reintroducing the electric field generated by the displacement currents. Furthermore, in Section III, we propose a modified discrete complex images approximation that leads us to analytical expressions for the Green's function.

In Section IV, we present the computation of the impedance matrix. For the short separations that are relevant to loopimpedance computations, we use a superposition principle that is applied to source dipoles, leading to analytical expressions for the self-impedance and the mutual-impedance between conductor loops under the modified discrete complex images approximation. Finally, in Section V, we compare the accuracy of our results with industry standard electromagnetic field solvers and show the computational efficiency of the substrate Green's function approach. In brief, we present a new analytical formulation to compute the loop-impedance matrix for general VLSI interconnect configurations in the presence of a multilayer conductive substrate. The maximum deviation of our results compared with FastHenry [4] is less than 3\% for realistic interconnect configurations. Concluding remarks are given in Section VI.

\section{BACKGROUND}

The 2-D quasi-static Green's function for an elementary excitation consisting of a single monopole current in the

\footnotetext{
${ }^{1}$ Generalization to passive devices such as inductors demands a 3-D treatment. Work is in progress in that direction.
} 


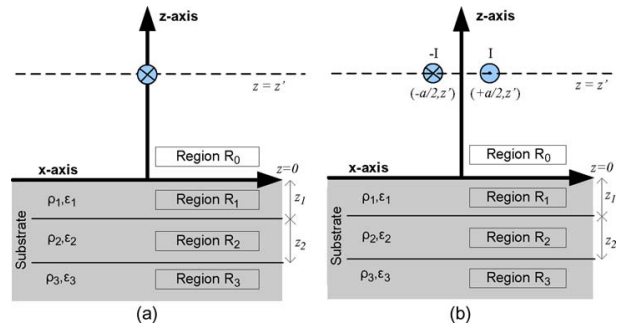

Fig. 3. Cross-sectional schematic of (a) an open line current located at $\left(0, z^{\prime}\right)$ and (b) a 2-D magnetic dipole formed by two parallel opposing line currents $-I$ and $I$ lying above a three-layer substrate. The third layer (region $R_{3}$ ) extends to $z=-\infty$.

presence of a stratified substrate has been discussed in [6]. The configuration is shown in Fig. 3(a), where a single-line current (monopole source) at coordinates $\left(x^{\prime}, z^{\prime}\right)$ lies above a three-layer conductive substrate. Translational invariance of the Green's function permits us to place the source at $x^{\prime}=0$ without any loss of generality.

The monopole Green's function $G_{0, \text { mono }}\left(x, z, x^{\prime}, z^{\prime}\right)$ for the magnetic vector potential $A(x, z)$ in region $R_{0}$ (above the substrate) satisfies

$$
\begin{aligned}
\nabla^{2} G_{0, \text { mono }}\left(x, z, x^{\prime}, z^{\prime}\right) & =-\mu \delta\left(x-x^{\prime}\right) \delta\left(z-z^{\prime}\right), \\
x^{\prime} & =0, \quad z \geq 0 .
\end{aligned}
$$

Since there are no sources inside the substrate layers (regions $R_{i}, i>0$ ), each of which has conductivity $\sigma_{i}$, permittivity $\epsilon_{i}$, and permeability $\mu$ (relative permeability of all regions $\mu_{r} \approx 1$ ), the Green's functions in these regions satisfy

$$
\begin{aligned}
\nabla^{2} G_{i}\left(x, z, x^{\prime}, z^{\prime}\right) & -j \omega \mu\left(\sigma_{i}+j \omega \epsilon_{i}\right) \\
& \times G_{i}\left(x, z, x^{\prime}, z^{\prime}\right)=0, i>0, \quad z \leq 0 .
\end{aligned}
$$

Solving (1) and (2) using the boundary conditions

$$
\left(\vec{B}_{R_{i}}-\vec{B}_{R_{i-1}}\right) \cdot \hat{z}=0 ; \quad\left(\vec{H}_{R_{i}}-\vec{H}_{R_{i-1}}\right) \times \hat{z}=0
$$

at the interface boundaries between different regions results in the Fourier integral expression for $G_{0, \text { mono }}\left(x, z, x^{\prime}, z^{\prime}\right)$, which has been derived elsewhere [6], [7], [13], [14], i.e.,

$$
\begin{aligned}
G_{0, \text { mono }}\left(x, z, 0, z^{\prime}\right)= & -\frac{\mu}{2 \pi} \times \int_{0}^{\infty}\left(e^{-\left|z-z^{\prime}\right| \lambda} \frac{\cos (\lambda x)}{\lambda}\right. \\
& \left.-g_{N}(\lambda) e^{-\left(z+z^{\prime}\right) \lambda} \frac{\cos (\lambda x)}{\lambda}\right) d \lambda .
\end{aligned}
$$

The function $g_{N}(\lambda)$, which characterizes the substrate contribution for an $N$-layer substrate, has the functional form

$$
g_{N}(\lambda)=\frac{Q_{N}(\lambda)-\lambda}{Q_{N}(\lambda)+\lambda}
$$

where the terms $Q_{N}(\lambda)$ are easily derived and are dependent on the properties $(\sigma, \epsilon, \mu)$ of each substrate layer. For the simplest case of a one-layer substrate $(N=1)$ extending to $z=-\infty$ (occupying a half-space)

$$
Q_{1}(\lambda)=\sqrt{\lambda^{2}+\gamma_{1}^{2}}
$$

The quantity $\gamma_{1}^{2}=j \omega \mu\left(\sigma_{1}+j \omega \epsilon_{1}\right)$ depends on the frequency $\omega$ and substrate properties-permeability $\mu$, resistivity $\rho=$ $1 / \sigma_{1}$, and permittivity $\epsilon_{1}$.
TABLE I

SubSTRATE RESISTIVITY VALUES AND CORRESPONDING SKIN DEPTH AT 50-GHz FREQUENCY

\begin{tabular}{|l|c|c|c|c|}
\hline Resistivity $(\Omega-c m)$ & 0.01 & 0.1 & 1 & 10 \\
\hline Skin depth $(\mu m)$ & 22.5 & 71.2 & 225 & 711 \\
\hline
\end{tabular}

The first term in the integrand in (4), which represents the source contribution in free space, is analytically integrable. The second term, which represents the substrate contribution and involves $g_{N}(\lambda)$, cannot be integrated analytically. Several techniques have been proposed to compute the Green's function (4), which we briefly review in Section II-A.

\section{A. Computation of the Substrate Green's Function}

A straightforward approach to evaluate the substrate Green's function is to use numerical integration techniques [14]. However, this approach is computationally too expensive to handle complex VLSI interconnect configurations that typically consist of millions of wires. Alternative approaches to compute this Green's function, as discussed in the following paragraphs, approximate the term $g_{N}(\lambda)$ in (4) with suitable expressions that make the substrate contribution to the Green's function analytically integrable.

1) Approximate Complex Image Method (ACIM): In this approach [8], $g_{1}(\lambda)$, for a one-layer substrate, is approximated by its Taylor series expansion around $\lambda=0$, leading to the expression [6]

$$
\frac{\sqrt{\lambda^{2}+\gamma_{1}^{2}}-\lambda}{\sqrt{\lambda^{2}+\gamma_{1}^{2}}+\lambda}=e^{-2 \lambda / \gamma_{1}}\left(1+\frac{\lambda^{3}}{3 \gamma_{1}^{3}}+\cdots\right) .
$$

Keeping only the first term in the series expansion, (4) becomes

$$
\begin{aligned}
& G_{0, \text { mono }}\left(x, z, 0, z^{\prime}\right) \\
& \quad \approx-\frac{\mu}{2 \pi} \int_{0}^{\infty}\left(e^{-\left|z-z^{\prime}\right| \lambda}-e^{-\left(z+z^{\prime}+\frac{2}{\gamma_{1}}\right) \lambda}\right) \frac{\cos (\lambda x)}{\lambda} d \lambda .
\end{aligned}
$$

The expression in (8) is analytically integrable. The first exponential term arises from the source (line current) at point $\left(0, z^{\prime}\right)$, whereas the second one represents the effect of an opposing current (negative sign) lying at point $\left(0,-z^{\prime}-2 / \gamma_{1}\right)$. In other words, the effect of the substrate is represented in this approximation by a single image of the source line current. The image is located "inside" the substrate at a depth given by a complex number, hence the name "complex image." This method has been applied to VLSI interconnect extraction [6], [7], [15].

However, the above approximation is valid only when the separation of the observation point $(x, z)$ from the source at $\left(0, z^{\prime}\right)$ is much larger than the skin depth $[\delta=\sqrt{2 /(\omega \mu \sigma)}]$ inside the substrate [8]. Table I indicates that the micrometersize separations of interest for VLSI interconnect impedance extraction are much smaller than the skin depth at $50 \mathrm{GHz}$ for realistic values of substrate resistivity. Hence, this method, although suitable for computing the interaction of antennas with the earth (substrate), is not suitable for VLSI interconnects.

2) Rational Function Fit Method (RFFM): An alternative method for evaluating the substrate Green's function involves 
approximating it by a rational function that can be integrated analytically [9]. The rational function fit can uniquely be defined in terms of a set of $K$ pole-residue pairs $\left(A_{n}, C_{n}\right)$ :

$$
\int_{0}^{\infty} g_{N}(\lambda) e^{-\left(z+z^{\prime}\right) \lambda} \frac{\cos (\lambda x)}{\lambda} d \lambda \cong \int_{0}^{\infty} \sum_{n=1}^{K} \frac{C_{n}}{j \lambda^{2}-A_{n}} d \lambda .
$$

The pole-residue extraction demands a nonlinear least square fit for every point $(x, z)$ in space and is computationally expensive. Achieving accuracy within $1 \%-2 \%$ using this technique often requires a number of poles $K$ on the order of 100 for each $(x, z)$ point. The number of such points $(x, z)$ must also be large. For these reasons, although this methodology can be applied for parasitic extraction of interconnects, it is computationally expensive and memory intensive.

3) DCIM: The discrete complex images method (DCIM) [16] can be considered as an extension to the approximate complex image method (ACIM), which approximates $g_{N}(\lambda)$ using a number of complex images:

$$
g_{N}(\lambda) \approx \sum_{j=1}^{M} b_{j} e^{-c_{j} \lambda}
$$

where each $j$ th exponential term represents an image, and $\left(b_{j}, c_{j}\right) \in \mathbb{C}$. Both $M$ and $\left(b_{j}, c_{j}\right)$ are the variables to be adjusted. The resultant integral has a similar form as that shown in (8) and can be evaluated analytically. The DCIM is the method of choice for evaluating the 3-D full-wave Green's functions for layered media [17], [18], which are known as Sommerfeld layered-media Green's functions [19]. However, finding a set of complex images $\left(b_{j}, c_{j}\right)$ that result in an accurate approximation to the Green's function remains a challenge [20], [21]. The DCIM has been applied to VLSI interconnect impedance extraction in FastMaxwell [22] for single-layer substrate configurations.

\section{GREen's FunCtion FOR A MAGNetic Dipole IN THE Presence of a CONDUCTIVE Substrate}

The magnetic monopole Green's function leads to a PEEC [5] approach that has an unphysical long-distance behavior, with the inductance per unit length logarithmically decaying with wire separation, instead of the correct power law decay associated with closed current (loop) approaches [10]. The end result is dense and nondiagonal-dominant impedance matrices. In this paper, we are interested in computing physically measurable quantities, i.e., in terms of loop quantities, resulting in sparse impedance matrices.

We start from a Green's function corresponding to a closed elementary loop, i.e., a magnetic dipole. It has the correct longdistance behavior for the magnetic field and the correct lowfrequency behavior corresponding to MQS phenomena. It can be verified that it is the quasi-static limit of the Sommerfeld layered-media Green's function.

Fig. 3(b) schematically shows a vertical magnetic dipole in two dimensions (two parallel lines carrying opposing currents forming a current loop) above a three-layer substrate. The dipole is centered at $\left(x^{\prime}=0, z^{\prime}\right)$ and is directed normal to the plane containing the two currents. The two line currents $-I$

and $I$ are located at $\left(-a / 2, z^{\prime}\right)$ and $\left(+a / 2, z^{\prime}\right)$, respectively, such that the diameter of the dipole is $a$. The magnetic vector potential $A(x, z)$ due to this dipole satisfies the relation

$$
\begin{aligned}
& \nabla^{2} A_{0, \text { dipole }}^{\mathrm{ver}}\left(x, z, x^{\prime}, z^{\prime}\right) \\
& =-\mu I\left(\delta\left(x+\frac{a}{2}\right) \delta\left(z-z^{\prime}\right)-\delta\left(x-\frac{a}{2}\right) \delta\left(z-z^{\prime}\right)\right) \\
& =-\mu(I a) \frac{\delta\left(x+\frac{a}{2}\right) \delta\left(z-z^{\prime}\right)-\delta\left(x-\frac{a}{2}\right) \delta\left(z-z^{\prime}\right)}{x^{\prime}=0 \quad z \geq 0 .}
\end{aligned}
$$

A dipole is defined as the limit of the configuration in Fig. 3(b) when $a \rightarrow 0$ and $I \rightarrow \infty$, such that the dipole moment per unit length

$$
p=\frac{\mu}{2 \pi} I a
$$

is constant [23]. Hence, the Green's function for the magnetic vector potential $A_{0, \text { dipole }}^{\mathrm{ver}}$ due to a unitary vertical magnetic dipole $(p=1)$ satisfies the relation

$$
\begin{aligned}
& \nabla^{2} G_{0, \text { dipole }}^{\mathrm{ver}}\left(x, z, x^{\prime}=0, z^{\prime}\right) \\
& =-2 \pi \lim _{a \rightarrow 0} \frac{\delta\left(x+\frac{a}{2}\right) \delta\left(z-z^{\prime}\right)-\delta\left(x-\frac{a}{2}\right) \delta\left(z-z^{\prime}\right)}{a} .
\end{aligned}
$$

Since there are no sources in the substrate, (2) remains valid. Using continuity at the interface boundaries between different regions, (13) for a unitary magnetic dipole situated at $\left(0, z^{\prime}\right)$ gives

$$
\begin{aligned}
& G_{0, \text { dipole }}^{\text {ver }}\left(x, z, x^{\prime}=0, z^{\prime}\right) \\
& =-\lim _{a \rightarrow 0} \frac{\int_{0}^{\infty}\left(e^{-\left|z-z^{\prime}\right| \lambda}-g_{N}(\lambda) e^{-\left(z+z^{\prime}\right) \lambda}\right) \frac{2 \sin (\lambda x) \sin \left(\lambda \frac{a}{2}\right)}{\lambda} d \lambda}{a} \\
& =-\int_{0}^{\infty}\left(e^{-\left|z-z^{\prime}\right| \lambda}-g_{N}(\lambda) e^{-\left(z+z^{\prime}\right) \lambda}\right) \sin (\lambda x) d \lambda
\end{aligned}
$$

The Green's function for a horizontal magnetic dipole can be derived in similar fashion, i.e.,

$$
\begin{aligned}
& G_{0, \text { dipole }}^{\text {hor }}\left(x, z, x^{\prime}=0, z^{\prime}\right) \\
& \quad=-\int_{0}^{\infty}\left(e^{-\left|z-z^{\prime}\right| \lambda}+g_{N}(\lambda) e^{-\left(z+z^{\prime}\right) \lambda}\right) \cos (\lambda x) d \lambda .
\end{aligned}
$$

In general, $g_{N}(\lambda)$, which is the term characterizing the substrate contribution for an $N$-layer substrate, can be cast into the form shown in (5). The corresponding $Q_{N}(\lambda)$ functions become increasingly more complex as we increase the number of layers. For a single-layer substrate, $Q_{1}(\lambda)$ satisfies (6). The corresponding expressions for two- and three-layer substrates are

$$
\begin{aligned}
Q_{2}(\lambda) & =m_{1} \times \frac{\left(m_{1}+m_{2}\right) e^{2 m_{1} z_{1}}-\left(m_{1}-m_{2}\right)}{\left(m_{1}+m_{2}\right) e^{2 m_{1} z_{1}}+\left(m_{1}-m_{2}\right)} \\
Q_{3}(\lambda) & =m_{1} \times \frac{1-e^{-2 m_{1} z_{1}} q(\lambda)}{1+e^{-2 m_{1} z_{1}} q(\lambda)}, \text { with } \\
q(\lambda) & =\frac{\left[\begin{array}{c}
\left(m_{1}+m_{2}\right)\left(m_{2}-m_{3}\right) \\
\left.+\left(m_{1}-m_{2}\right)\left(m_{2}+m_{3}\right) e^{2 m_{1}\left(z_{2}-z_{1}\right)}\right]
\end{array}\right.}{\left[\begin{array}{c}
\left(m_{1}+m_{2}\right)\left(m_{2}+m_{3}\right) \\
\left.+\left(m_{1}-m_{2}\right)\left(m_{2}-m_{3}\right) e^{2 m_{1}\left(z_{2}-z_{1}\right)}\right]
\end{array}\right.}
\end{aligned}
$$


In (16) and (17), $z_{1}$ refers to the thickness of the top substrate layer, and $z_{2}$ refers to the thickness of the second substrate layer (in the case of a three-layer substrate), whereas the last substrate layer extends to $-\infty$, as shown in Fig. 3(b). The coefficients $m_{i}$, which correspond to the $i^{\text {th }}$ substrate layer, are given by

$$
m_{i}(\lambda)=\sqrt{\lambda^{2}+\gamma_{i}^{2}}=\sqrt{\lambda^{2}+j \omega \mu\left(\sigma_{i}+j \omega \epsilon_{i}\right)} .
$$

Typical semiconductor chip substrates have two or three layers with different resistivity values [2]. Hence, the expressions presented so far encompass the relevant scenarios to characterize realistic process configurations. Next, we propose an accurate analytical approximation to the dipole Green's function in the presence of multilayer substrates.

\section{A. Modified DCIM}

In (14) and (15), the term containing $g_{N}(\lambda)$ prevents an analytical expression for the Green's function. Using the DCIM, $g_{N}(\lambda)$ can be approximated by a sum of complex exponentials (10), leading to the desired integrable form of the substrate Green's function. The main difficulty with the DCIM is the search for a suitable set of "complex images," $c_{j}$, that gives accurate results. Our objective is to simplify the search for these complex exponentials.

We start with a new look at the form of the dipole Green's function in the presence of a substrate. For a single-layer substrate, with $Q_{1}(\lambda)$ given by (6), we can perform the following algebraic replacement:

$$
\begin{aligned}
g_{1}(\lambda) & =e^{-2 \tanh ^{-1}\left(\lambda / \sqrt{\lambda^{2}+\gamma_{1}^{2}}\right)} \\
& =e^{-2 \tanh ^{-1}\left(\left(\lambda / \gamma_{1}\right) / \sqrt{1+\left(\lambda / \gamma_{1}\right)^{2}}\right)} \\
& =e^{-2 \sinh ^{-1}\left(\frac{\lambda}{\gamma_{1}}\right) .}
\end{aligned}
$$

This alternative exponential representation of $g_{1}(\lambda)$ naturally leads us to a separation of the coefficient $1 / \gamma_{1}$, which constitutes the sole complex part of the exponent. The separation of this complex term allows us to approximate the expression $g_{1}(\lambda)$ using real coefficients $\alpha_{k}$. This is in contrast with the DCIM, which involves complex coefficients $c_{j}$. Furthermore, we add coefficients $\beta_{k}$ to perform a linear combination of the complex exponentials and propose the following approximation:

$$
g_{1}(\lambda) \approx \sum_{k=1}^{K} \beta_{k} e^{-\alpha_{k}\left(\lambda / \gamma_{1}\right)}, \quad \alpha_{k} \in \mathbb{R}, \quad \beta_{k} \in \mathbb{C} .
$$

Physically, approximation (20) has a similar interpretation to that of the DCIM. The $k^{\text {th }}$ term in the approximation constitutes an image of the dipole current source whose $z$-displacement is dependent on $\alpha_{k}$, as shown in Fig. 4, whereas the coefficient $\beta_{k}$ modifies the magnitude of the image current.

From (20), it is clear that $\alpha_{k}>0$ ensures convergence. Moreover, since $g_{1}(0)=1$, it follows that $\sum_{k} \beta_{k}=1+j 0$. The nonlinear fitting algorithm we use finds an accurate approximation with the parameters $\left(\alpha_{k}, \beta_{k}\right)$ that naturally satisfy these properties. We use MATLAB's built-in function "fminsearch" to determine the best fit to parameters $\alpha_{k}$ and $\beta_{k}$. The starting

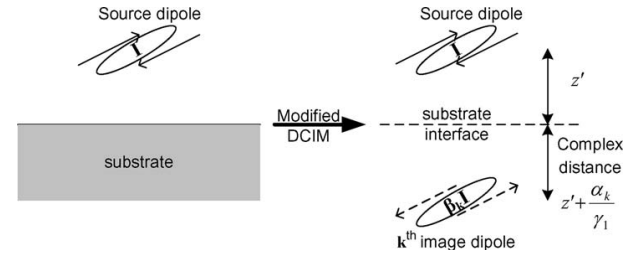

Fig. 4. Physical interpretation of the modified DCIM.

value of all parameters is set to 1 . Since $\alpha_{k} \in \mathbb{R}$, the search space for the set of "complex images" is smaller.

The number of images $K$ determines the computational expense in finding the set of images $\left(\alpha_{k}, \beta_{k}\right)$ and the accuracy of the approximation for a given substrate configuration. Large values of $K$ lead to instabilities, making it difficult to find a good fit. In our experiments, we found that $K=5$ gave the desired accuracy for the impedance (within $2 \%$ of the field solver) while keeping the computation time for the set of images $\left(\alpha_{k}, \beta_{k}\right)$ within a few minutes.

Inserting (20) into (14) and (15), we obtain the following analytical expressions for the Green's functions:

$$
\begin{aligned}
& G_{0, \text { dipole }}^{\mathrm{ver}}\left(x, z, z^{\prime}\right) \\
& \quad \approx-\int_{0}^{\infty}\left(e^{-\left|z-z^{\prime}\right| \lambda}-\sum_{k=1}^{K} \beta_{k} e^{-\alpha_{k} \frac{\lambda}{\gamma_{1}}} e^{-\left(z+z^{\prime}\right) \lambda}\right) \sin (\lambda x) d \lambda \\
& \quad=-\left[\frac{x}{\left|z-z^{\prime}\right|^{2}+x^{2}}-\sum_{k=1}^{K}\left(\beta_{k} \frac{x}{\left(z+z^{\prime}+\frac{\alpha_{k}}{\gamma_{1}}\right)^{2}+x^{2}}\right)\right] \\
& G_{0, \text { dipole }}^{\text {hor }}\left(x, z, z^{\prime}\right) \\
& \approx-\int_{0}^{\infty}\left(e^{-\left|z-z^{\prime}\right| \lambda}+\sum_{k=1}^{K} \beta_{k} e^{-\alpha_{k} \frac{\lambda}{\gamma_{1}}} e^{-\left(z+z^{\prime}\right) \lambda}\right) \cos (\lambda x) d \lambda \\
& \quad=-\left[\frac{\left|z-z^{\prime}\right|}{\left|z-z^{\prime}\right|^{2}+x^{2}}+\sum_{k=1}^{K}\left(\beta_{k} \frac{z+z^{\prime}+\frac{\alpha_{k}}{\gamma_{1}}}{\left(z+z^{\prime}+\frac{\alpha_{k}}{\gamma_{1}}\right)^{2}+x^{2}}\right)\right] .
\end{aligned}
$$

We use the same replacement (20) for the two- and threelayer substrate configurations. Hence, for the general $N$-layer substrate

$$
g_{N}(\lambda) \approx \sum_{k=1}^{K} \beta_{k} e^{-\alpha_{k}\left(\lambda / \gamma_{1}\right)}, \quad \alpha_{k} \in \mathbb{R}, \quad \beta_{k} \in \mathbb{C}
$$

and expressions (21) and (22) remain valid. $\alpha_{k}$ and $\beta_{k}$ are parameters that depend only on the substrate profile (resistivity, dielectric constant, and thickness of each layer) and the frequency of interest. Hence, the computation of these parameters constitutes a one-time cost for a given technology at each frequency.

\section{B. Accuracy of the Modified DCIM and Comparison With Other Methods}

Figs. 5(a) and (b) show that (20) provides a good approximation to the numerically computed value of $g_{1}(\lambda)$. The same approximation using the ACIM shows significant deviation. Given the oscillatory nature of the Green's function, the 
(a)

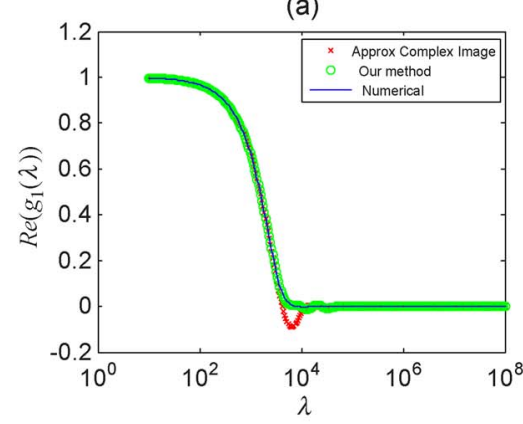

(b)

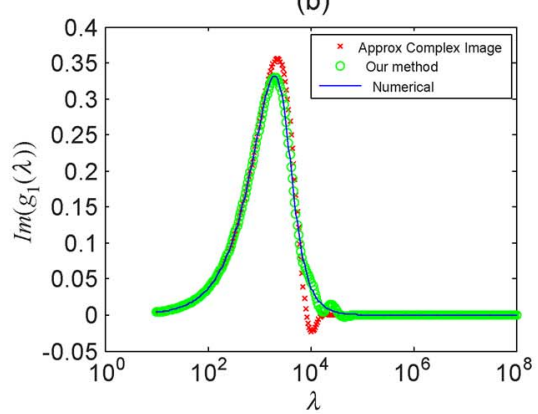

(c)

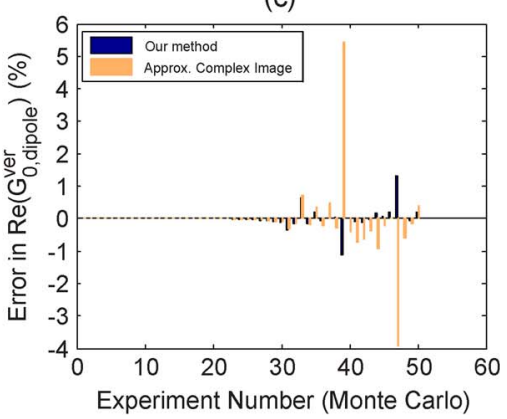

Fig. 5. Approximation of the (a) real and (b) imaginary parts of $g_{1}(\lambda)$ using our method (20) (with $K=20$ ) and the ACIM for a one-layer substrate, $\rho=1 \Omega \cdot \mathrm{cm}$. (c) Monte Carlo results for error in the substrate Green's function using our method (21) and the ACIM, with respect to numerical computation. Monte Carlo simulations are done for frequency $20-100 \mathrm{GHz}, z$-separation $0.5-1.0 \mu \mathrm{m}$, and $x$-separation $0.5-10 \mu \mathrm{m}$.

(a)

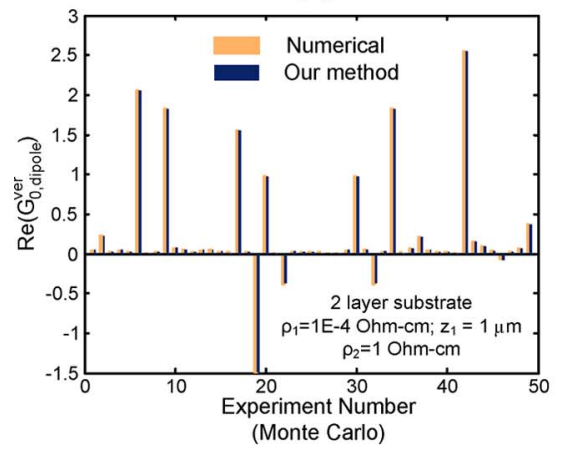

(b)

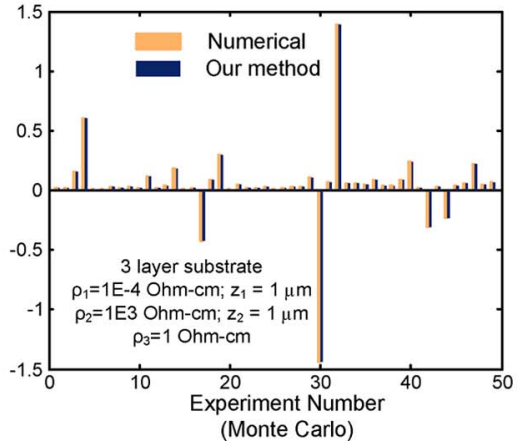

(c)

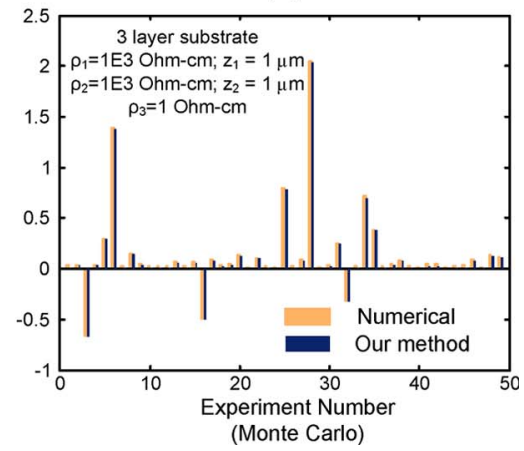

Fig. 6. Monte Carlo simulations comparing the proposed approximation (21) of the substrate Green's function to the numerical evaluation of (14) for (a) a two-layer substrate with $K=20$ images, and [(b) and (c)] a three-layer substrate with two different profiles with $K=5$ images. Frequency $20-100$ GHz, $z$-separation $0.5-1.0 \mu \mathrm{m}, x$-separation $0.5-10 \mu \mathrm{m}$.

$x$ - and $z$-separations are critical factors affecting the quality of the approximation besides the frequency and substrate parameters. We perform Monte Carlo simulations to evaluate the substrate Green's function $G_{0 \text {,dipole }}^{\text {ver }}(21)$ over a wide range of frequencies and $x$ - and $z$-separations. Fig. 5(c) plots the resulting error in the Green's function computation for a typical one-layer substrate. While the error using our method is always small, the ACIM sometimes gives much larger errors. This is because the ACIM is not valid for all $x$ - and $z$-separations and frequencies, as explained in Section II. The cases with small errors are those in which the randomly chosen geometries are such that the effect of the substrate is small, and the error in the ACIM is not reflected in the overall Green's function.

The Monte Carlo results for typical two- and three-layer substrates are shown in Fig. 6. In all cases, the analytical expression for the substrate Green's function shown in (21) provides an excellent agreement with the numerical calculation. Similar results are obtained for (22).

Although several techniques have been proposed to implement the DCIM to approximate the 3-D substrate Green's function [20], [24]-[27], it still remains a challenging task [20], [21]. Two features have been widely mentioned as limitations of the DCIM in the literature [20], [28]. The first is the use of a uniform sampling rate in the spectrum variable $\lambda$, leading to either very fine sampling in $\lambda$ or different DCIM parameterizations, to capture steep variations in the fitted function $g_{N}(\lambda)$ for typical multilayer substrates. The second is its sensitivity to the signal frequency interval.
To implement the DCIM for the 2-D quasi-static Green's function, we use the variable projection method [29], which has been widely used for exponential fitting problems [30]. The sampling points in $\lambda$ are user-defined, giving wide latitude for nonuniform sampling to account for the rapid variations in $g_{N}(\lambda)$. Moreover, the linear coefficients $\left[b_{j}\right.$ in (10) or $\beta_{k}$ in (23)] are uniquely determined once a solution to the nonlinear parameter least square problem is found. Hence, random starting values are needed only for the nonlinear parameters $\left[c_{j}\right.$ in (10) or $\alpha_{k}$ in (23)]. The residual errors and the computation time to perform the two fits (10) and (23) are compared in Fig. 7(a) and (b) as a function of the number of complex images. It is found that our method converges to an optimal solution faster and with greater accuracy than the DCIM. Fig. 7(c) shows that the results from our method are smooth as a function of frequency, a property not shared by the DCIM. Finally, Fig. 8 compares the Green's function computation using the DCIM and our method for the same number of complex images. The superior performance of our method stems from the fact that the search for exponential fitting parameters, which is in unconstrained complex space under the DCIM, is constrained to a search for real coefficients applied to a specific complex number $\left[1 / \gamma_{1}\right.$ in (23)] under our method.

In comparison with the rational function fit method (RFFM), the advantage of our method is in terms of computation cost. Computing the Green's function $G\left(x, z, x^{\prime}, z^{\prime}\right)$ using the RFFM involves the evaluation of the pole-residue pairs in (9) for each pair of points $(x, z)$ and $\left(x^{\prime}, z^{\prime}\right)$. Impedance extraction of a 
(a)

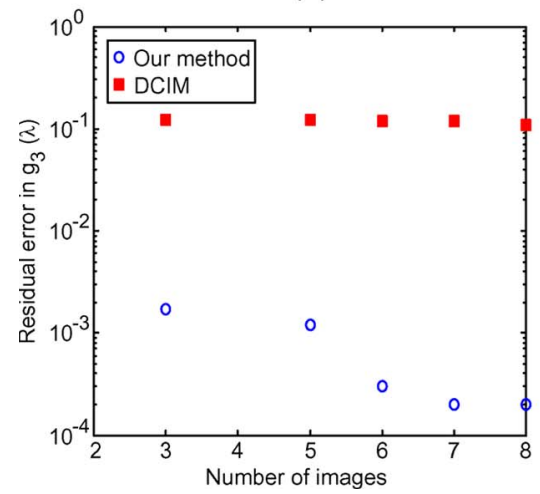

(b)

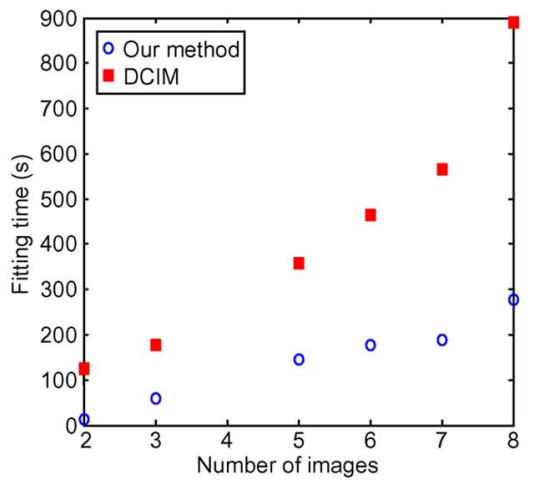

(c)

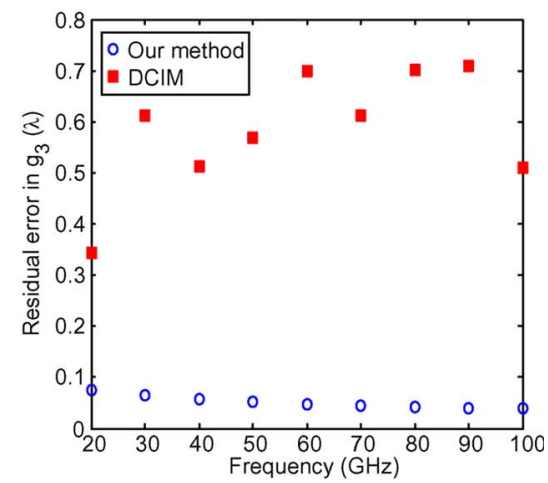

Fig. 7. (a) Residual error and (b) time for computing the complex image approximation using the DCIM and our method for a three-layer substrate at 90 GHz. (c) Residual error in the complex image approximation using the DCIM and our method for a three-layer substrate with five images as a function of frequency.

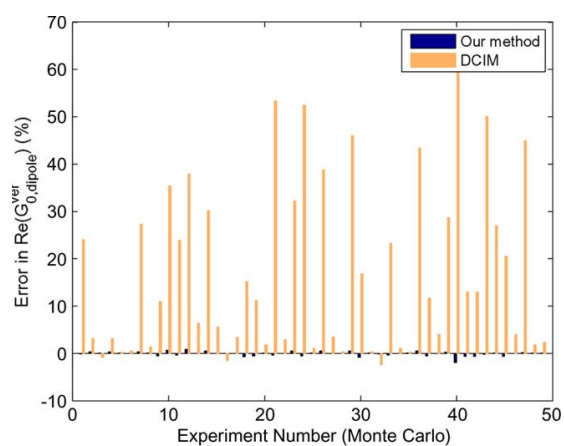

Fig. 8. Error in substrate Green's function computation (21) using the DCIM and our method with respect to the numerical computation for a three-layer substrate. Number of images $M=K=5$. Monte Carlo simulations are done for frequency $20-100 \mathrm{GHz}, z$-separation $0.5-1.0 \mu \mathrm{m}$, and $x$-separation $0.5-10 \mu \mathrm{m}$.

TABLE II

Cost of Computing One Element of THE LOOP-IMPEDANCE MATRIX With THE RFFM AND With OUR METHOd IN THE PRESENCE OF a Two-Layer Substrate. $T_{i}$ : Time to EXtract a Set of

Complex Images (One-Time Cost); $T_{p}$ : Time to Compute One Set of Pole-Residue Fits; $n_{\text {fits }}$ : NuMBER of POLE-RESIDUE FITS NEEDED PER $Z$-MATRIX ElEMENT (USING THE DDA [31]); $T_{Z}$ : Time to Compute One $Z$-MATRiX Element

\begin{tabular}{|l|c|c|c|c|}
\hline & $T_{i}(\mathrm{~s})$ & $T_{p}(\mathrm{~s})$ & $n_{\text {fits }}$ & $T_{Z}(\mathrm{~s})$ \\
\hline RFFM & 0 & 16.9 & 40 & 674 \\
Our method & 91.34 & 0 & 0 & $<1 \mathrm{sec}$ \\
\hline
\end{tabular}

general interconnect configuration requires numerous computations of $G$ at coordinates that are not known a priori. In Table II, we show the computation time for one element of the loop-impedance matrix shown in (34). Here, we have used the discrete dipole approximation (DDA) [31] with ten discrete horizontal and vertical dipoles each, resulting in $10 \times 2 \times 2=$ 40 computations for each impedance matrix element. Since the impedance matrix for a realistic interconnect configuration may require hundreds of such computations (the interconnect shown in Section V with 15 filaments per conductor requires $15^{2}=$ 225 such computations), the cost of using the RFFM quickly becomes prohibitive. On the other hand, with our method, the complex exponential fit does not need to be repeated for every interconnect configuration. Hence, although the computation time for each fitting step with the RFFM may be reduced by trading off accuracy, this will not bridge the orders-of- magnitude gap in impedance computation expense between the RFFM and our method.

\section{INTERCONNECT IMPEDANCE COMPUTATION USING THE GREEN'S FunCTION FOR A MAGNETIC DiPOLE}

We now address the problem of computing interconnect impedance for a set of interacting conductors in the presence of a multilayer conductive substrate. Since we solve the 2-D problem, the conductors are assumed to have a common length $L$. The general impedance extraction problem is schematically shown in Fig. 1, where Loop1 denotes the source of the magnetic field, and Loop2 denotes the victim conductor loop. The self-impedance of each loop and the mutual impedance between the two loops in the presence of the multilayer substrate are sought. This section presents new analytical expressions to compute these impedances. The magnetic vector potential $A(x, z)$ due to the source conductor loop is first computed in Section IV-A. These expressions for $A(x, z)$ are valid for all separations. In Section IV-B, the mutual- and selfimpedance computation using these analytical expressions is explained for conductors with cross-sectional dimensions that are small enough to be represented as single filaments. Finally, Section IV-C discusses the computation for wide/thick conductors, which we discretize into multiple filaments to capture the internal skin effects.

\section{A. Magnetic Vector Potential Due to a Finite-Size Conductor Loop}

Green's functions (21) and (22) give the magnetic vector potential at point $(x, z)$ due to a unitary magnetic dipole $(p=$ 1) located at $\left(0, z^{\prime}\right)$. In the general case, the vector potential at $(x, z)$ due to a finite-size magnetic dipole is obtained by integrating the Green's function times the source dipole moment density $P\left(x^{\prime}, z^{\prime}\right)$ over the coordinates of the source, i.e.,

$$
A(x, z)=\int_{x^{\prime} z^{\prime}} G\left(x, z, x^{\prime}, z^{\prime}\right) P\left(x^{\prime}, z^{\prime}\right) d x^{\prime} d z^{\prime} .
$$

It has been shown in [10] that, when the separations between the source $\left(x^{\prime}, z^{\prime}\right)$ and the destination $(x, z)$ are larger than roughly five times the diameter of the source dipole [ $a$ in 


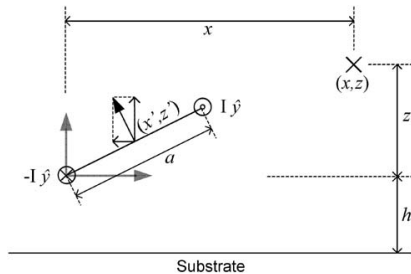

(a)

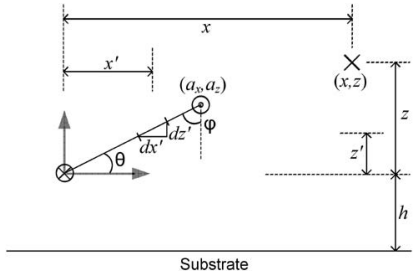

(b)
Fig. 9. (a) Source current loop represented as a single dipole at its centerDA - and resolved into horizontal and vertical components. This approximation is valid only at large distances from the source loop. (b) Source current loop represented as a continuous distribution of magnetic dipoles along the line joining the two ends of the conductor loop. This approximation is valid even at very short distances from the source loop.

Fig. 9(a)], the dipole can be approximated by a point source at its center. This is the "dipole approximation" (DA) [10]. Under this approximation, the magnetic vector potential is given by

$$
A_{\mathrm{DA}}(x, z)=G\left(x, z, x^{\prime}, z^{\prime}\right) \times \frac{\mu}{2 \pi} I a
$$

where $I$ denotes the current flowing through Loop1, $a$ is the diameter of the dipole (the distance between the two conductors forming the loop), and $p=(\mu / 2 \pi) I a$ is the dipole moment acting at point $\left(x^{\prime}, z^{\prime}\right)$, which lies at its center. This is schematically shown in Fig. 9(a). Since the vector potential resulting from both horizontal and vertical components of the dipole moment $p$ is directed along the third dimension $\hat{y}$, the value of the Green's function $G\left(x, z, x^{\prime}, z^{\prime}\right)$ in (25) is simply the algebraic sum of $G_{0 \text {,dipole }}^{\text {hor }}$ and $G_{0 \text {,dipole }}^{\text {ver }}$

However, for the impedance extraction problem, it is important to accurately compute the vector potential when separations are on the order of, or smaller than, the diameter of the source dipole. In this scenario, the DA is no longer valid, and the source must be replaced by a continuous distribution of magnetic dipoles along the line joining the two ends of the source current loop. As shown in Fig. 9(b), each infinitesimal magnetic dipole located at $\left(x^{\prime}, z^{\prime}\right)$ is resolved into its vertical and horizontal components with diameters $d x^{\prime}$ and $d z^{\prime}$ and dipole moments $(\mu / 2 \pi) I d x^{\prime}$ and $(\mu / 2 \pi) I d z^{\prime}$, respectively. The vector potential is then given by

$$
\begin{aligned}
A_{\text {cont }}(x, z)= & A_{\text {cont }}^{\text {ver }}(x, z)+A_{\text {cont }}^{\text {hor }}(x, z) \\
= & \int_{0}^{a_{x}} G_{0, \text { dipole }}^{\text {ver }}\left(x, z, x^{\prime}, z^{\prime}\right) \frac{\mu}{2 \pi} I d x^{\prime} \\
& +\int_{0}^{a_{z}} G_{0, \text { dipole }}^{\text {hor }}\left(x, z, x^{\prime}, z^{\prime}\right) \frac{\mu}{2 \pi} I d z^{\prime} .
\end{aligned}
$$

Employing (21) and (22), analytical expressions for $A_{\text {cont }}^{\text {ver }}$ and $A_{\text {cont }}^{\text {hor }}$ are obtained, and are shown in the Appendix. These expressions allow us to evaluate the magnetic vector potential at any point $(x, z)$ due to the (2-D) current loop formed by a pair of conductors in the presence of a multilayer substrate. A discretized form of the continuous dipole distribution in (26) has been presented in [31]. The analytical expressions derived here eliminate the need to compute the Green's function multiple times for a discrete set of dipoles, leading to a significantly more efficient computation.

\section{B. Impedance Extraction of Single-Filament Conductor Loops}

We now address the problem of mutual-impedance computation between a source magnetic dipole and the current loop formed by a pair of conductors with common length $L$. The mutual inductance per unit length can be computed as

$$
M=\frac{\psi}{I \cdot L}
$$

where $\psi$ denotes the flux due to the source magnetic field integrated over the area subtended by the victim conductor loop. The mutual inductance is a complex number [13]. The real part of $M$ captures the reactive losses due to the magnetic interaction between the source and the victim loop, whereas the imaginary part captures the resistive loss due to the eddy currents generated inside the substrate as well as in the victim loop. Accordingly, the mutual impedance $Z=j \omega M$ also has real and imaginary parts.

In (27), the flux linkage $\psi$ can be computed as

$$
\psi=\oint_{l} \vec{A}(\vec{r}) \cdot d \vec{l}
$$

where the contour integral is performed over the coordinates of the victim conductor loop.

For the 2-D problem considered here, the victim loop consists of the forward and return conductors, each of length $L$, where $L$ is much larger than the separation between them. Each of these conductors is located at a point in 2-D space (see Fig. 1), with its length oriented along the third dimension $\hat{y}$ [same as the direction of the vector potential $\vec{A}(\vec{r})$ ]. Hence, the integral in (28) reduces to

$$
\psi=A\left(r_{1}\right) \cdot L+A\left(r_{2}\right) \cdot(-L) .
$$

$A\left(r_{1}\right)$ and $A\left(r_{2}\right)$ can be computed by (26) using the analytical expressions shown in the Appendix. Substituting (29) in (27) gives

$$
M=\frac{\left(A\left(r_{1}\right)-A\left(r_{2}\right)\right) L}{I \cdot L}=\frac{A\left(r_{1}\right)-A\left(r_{2}\right)}{I} .
$$

Note that current $I$ in (26) and (30) cancels out, and, as expected, the resultant expression for inductance is a function of geometrical parameters only.

When computing the self-inductance of a loop, the victim conductor loop coincides with the source loop. In such cases, when the center-to-center distance between two conductors is zero, the correct way to compute the mutual impedance is to use the geometric mean distance (GMD) [32]. The GMD of a rectangular cross-sectional conductor with respect to itself is given by [32]

$$
d=e^{\log (w+t)-3 / 2}
$$

where $w$ and $t$ are the width and the thickness of the conductor filament, respectively. Hence, the loop self-inductance is given by

$$
\begin{aligned}
& M_{\text {self }}=\frac{A\left(s_{1}\right)-A\left(s_{2}\right)}{I} \\
& A\left(s_{1}\right)=A_{\text {cont }}^{\text {hor }}\left(d_{s_{1}}, 0\right)+A_{\text {cont }}^{\text {ver }}\left(0, d_{s_{1}}\right) \\
& A\left(s_{2}\right)=A_{\text {cont }}^{\text {hor }}\left(a_{x}+d_{s_{2}}, a_{z}\right)+A_{\text {cont }}^{\text {ver }}\left(a_{x}, a_{z}+d_{s_{2}}\right)
\end{aligned}
$$




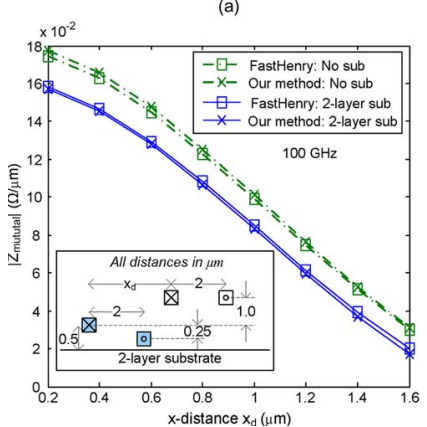

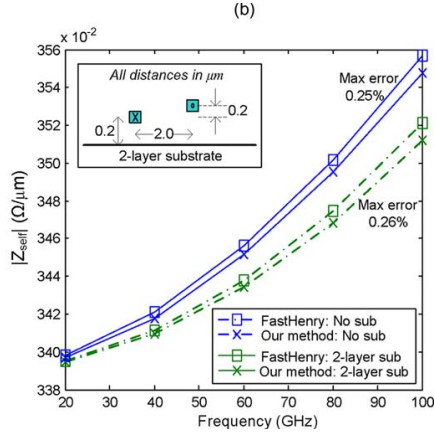

Fig. 10. (a) Mutual impedance $(Z=j \omega M)$ between (shaded) source current loop and (unshaded) victim current loop in the presence of a two-layer substrate for different separations $x_{d}$ at the highest frequency of concern $(100 \mathrm{GHz})$. (b) Self-impedance $\left|Z_{\text {self }}\right|$ of a conductor loop in the presence of a two-layer substrate as a function of frequency. The two-layer substrate configuration is the same as in Fig. 6(a); number of complex images $K=5$ for each conductor width $w=0.1 \mu \mathrm{m}$ and thickness $t=0.1 \mu \mathrm{m}$ (one filament per conductor).

where $d_{s_{1}}$ and $d_{s_{2}}$ are the GMDs corresponding to the filaments $s_{1}$ and $s_{2}$, respectively. The loop self-impedance is then given by

$$
Z_{\text {self }}=R_{\text {self }}+j \omega M_{\text {self }}
$$

where $R_{\text {self }}=\rho L\left(1 /\left(w_{s_{1}} t_{s_{1}}\right)+1 /\left(w_{s_{2}} t_{s_{2}}\right)\right)$ is the static resistance of the forward and return paths of the loop. As mentioned previously, another dynamic contribution to the resistance due to magnetic effects is embedded within $j \omega M_{\text {self }}$ as the imaginary part of $M_{\text {self }}$.

Fig. 10(a) shows the mutual impedance per unit length for different separations between two single-filament conductor loops, which is computed using the above analytical expressions compared with the field solver [4]. Fig. 10(b) shows that the self-impedance of a loop formed by a pair of conductors (with and without the substrate) calculated using the above analytical expressions has less than $1 \%$ error as compared with the field solver [4]. The conductor cross sections are chosen to allow a single-filament representation of each conductor. The results are accurate both in free space as well as in the presence of the substrate.

\section{Impedance Extraction of Realistic Multiple-Filament Conductor Bundles}

In case of wide/thick conductors with cross-sectional dimensions that are comparable to the skin depth at the frequency of concern, the computation is performed by dividing each conductor into filaments. Moreover, a signal wire may often have more than one nearby ground wire acting as its return path. We consider the collection of a signal wire (decomposed into multiple filaments $f_{1}$ to $f_{m}$ ) and its neighboring ground wires (decomposed into filaments $g_{1}$ to $g_{n}$ ) that constitute its return path. Every filament in the signal wire forms a loop with every filament in the corresponding return wires, resulting in $m \times n$ single-filament conductor loops. Such a collection of loops formed by the multiple filaments in a signal wire and its return paths is called a bundle. An example is shown in Fig. 11, where Bundle 1 has $D_{1}=m \times n$ loops, and Bundle 2 has $D_{2}=p \times q$ loops.

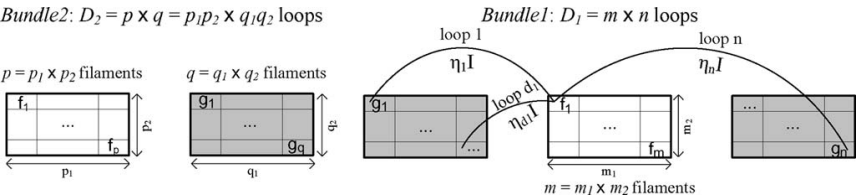

Fig. 11. Schematic showing wide and thick conductors discretized into multiple filaments. Bundle 1 has $m$ filaments in its signal wire and $n$ filaments in its two return wires, forming $D_{1}$ loops. Bundle 1 carries a total current $I$. Each $d_{1}^{\text {th }}$ loop in Bundle 1 carries a fraction $\eta_{d_{1}}$ of $I$. Bundle 2 has $p$ filaments in the signal and $q$ filaments in the return wire, forming $D_{2}$ loops.

The first step in computing the impedance of such bundles composed of multiple loops is to compute the loop-impedance matrix. The diagonal elements of matrix $Z_{\text {self }_{i}}$ are the loop self-impedances computed using (33). The off-diagonal terms $j \omega M_{i j}$ are the mutual impedances between loops computed using (30). Each $i^{\text {th }}$ loop in a bundle carries a fraction $\eta_{i}$ of the total current $I$ flowing through it. Once the loop-impedance matrix has been computed using the expressions derived previously, the coefficients $\eta_{i}$ can be found by solving the following Kirchhoff's equations:

$$
\begin{aligned}
\left.\begin{array}{ccc}
Z_{\text {self }_{1}} & j \omega M_{12} & \cdots \\
j \omega M_{21} & Z_{\text {self }_{2}} & \cdots \\
\vdots & \vdots & \ddots
\end{array}\right] & {\left[\begin{array}{c}
\eta_{1} I \\
\eta_{2} I \\
\vdots
\end{array}\right]=} \\
& \sum_{i} \eta_{i}=1 \quad \eta_{i} \in \mathbb{C} .
\end{aligned}
$$

From the system of equations (34), the loop self-impedance of a bundle can be found as $Z=I^{-1}$. For the two bundles shown in Fig. 11, index $d_{1}\left(d_{2}\right)$ runs over $D_{1}\left(D_{2}\right)$ constituent loops of Bundle1 (Bundle2). The loop self-impedance of Bundle1 (or Bundle2) and the fraction of current $\eta_{d_{1}}$ (or $\eta_{d_{2}}$ ) carried by each of its constituent loops can be found using (34).

The mutual impedance of Bundle 1 with respect to the $d_{2}^{\text {th }}$ loop in Bundle 2 can be expressed as

$$
M_{d_{2}}^{\text {Bundle } 1}=\sum_{d_{1}=1}^{D_{1}} \eta_{d_{1}} M_{d_{1}}^{d_{2}}
$$

where $M_{d_{1}}^{d_{2}}$ is the mutual impedance between the $d_{1}^{\text {th }}$ loop in Bundle 1 and the $d_{2}$ th loop in Bundle2. The computation of mutual impedances between these single-filament loops has been explained in Section IV-B. Finally, the net mutual impedance between Bundle 1 and Bundle 2 can be computed as

$$
M_{\text {Bundle } 2}^{\text {Bundle1 }}=\sum_{d_{2}=1}^{D_{2}} \eta_{d_{2}} M_{d_{2}}^{\text {Bundle1 }} .
$$

\section{RESULTS}

Here, we demonstrate the accuracy and the computational efficiency of our impedance extraction method, which is based on the 2-D MQS assumption, in the presence of a multilayer conductive substrate. The reader will note that the impedance computation involves integrals of a highly oscillatory Green's function, as shown in (14), and a further integral over the source dipole, as shown in (26). Both integrals may have significant cancellations depending on the interconnect geometry. Hence, it is not feasible to directly estimate the error 


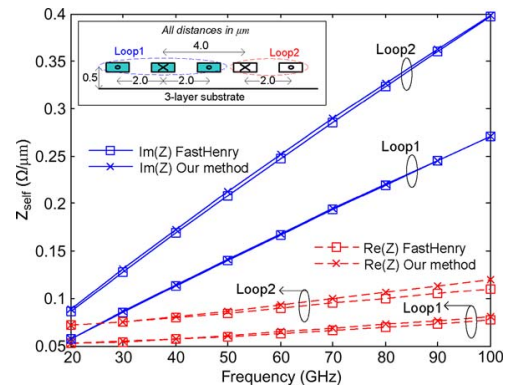

Fig. 12. Self-impedances of two multiple conductor bundles in the presence of a three-layer substrate. Each conductor has width $w=1 \mu \mathrm{m}$ and thickness $t=0.5 \mu \mathrm{m}(5 \times 3$ filaments per conductor $)$; number of complex images $K=5$. The maximum error in $\left|Z_{\text {self }}\right|$ is $1 \%$.

in the impedance computation from the error in the Green's function. To verify the accuracy of our approach, Section V-A shows the comparisons with the 3-D electromagnetic field solver FastHenry [4], as well as the commercial full-wave field solver High-Frequency Structure Simulator (HFSS) [33], for a wide range of realistic VLSI interconnect configurations. The computational efficiency of our method, due to the analytical expressions for the substrate Green's function, is shown in Section V-B through comparisons with 3-D (FastHenry) and with 2-D PEEC-based [5] computation. Note that, in all the examples shown, each conductor is discretized into the same number of filaments with our method as that with FastHenry or the PEEC to capture skin and proximity effects.

\section{A. Accuracy}

Fig. 12 shows the frequency-dependent loop self-impedance for two bundles composed of $1-\mu \mathrm{m}$-wide conductors in the presence of a three-layer substrate. The self-impedance computation shows a maximum $1 \%$ error in the magnitude of impedance in comparison with FastHenry across the entire frequency range.

When wide and thick conductors are discretized into multiple filaments to capture the skin and proximity effects, the relative positions of the filaments that comprise the conductor loops will not conform to the discrete positions occupied by conductors on individual metal layers. As an example of such a scenario, Fig. 13 shows that our method gives a small error $(<1.5 \%)$ for an arbitrary configuration of multiple conductors in the presence of a three-layer substrate.

Fig. 14(a)-(c) plots the self-impedance and the mutual impedance of two conductor loops on different metal layers. The vertical separation of the conductors above the substrate is chosen such that the effect of the substrate on the self-impedance and the mutual impedance is significant. The dashed lines in Fig. 14(d) show that the substrate causes as much as $20 \%$ variation in the impedance. It is found that our method captures the effect of the substrate on interconnect impedance with high accuracy (solid lines show errors less than 2\%) in the entire frequency range of interest for digital circuits $(20-100 \mathrm{GHz})$.

We now compare the network $Z$-parameter $Z_{\text {in }}$ for a conductor loop to that obtained from the commercial full-wave field solver HFSS [33]. The $Z_{\text {in }}$ parameter is computed by using a Spice distributed transmission line model, wherein the

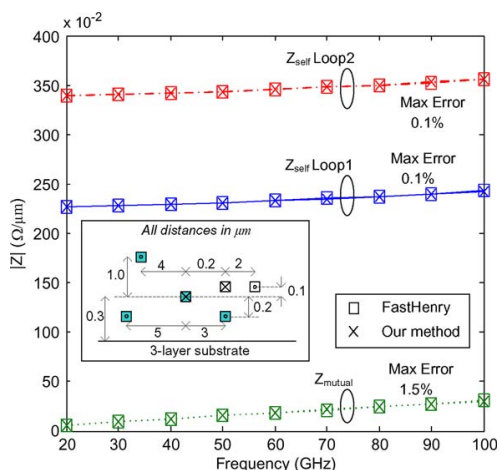

Fig. 13. Self-impedance and mutual impedance of two multiple conductor bundles in the presence of a three-layer substrate. Each conductor has width $w=0.1 \mu \mathrm{m}$ and thickness $t=0.1 \mu \mathrm{m}$ (one filament per conductor); number of complex images $K=5$.
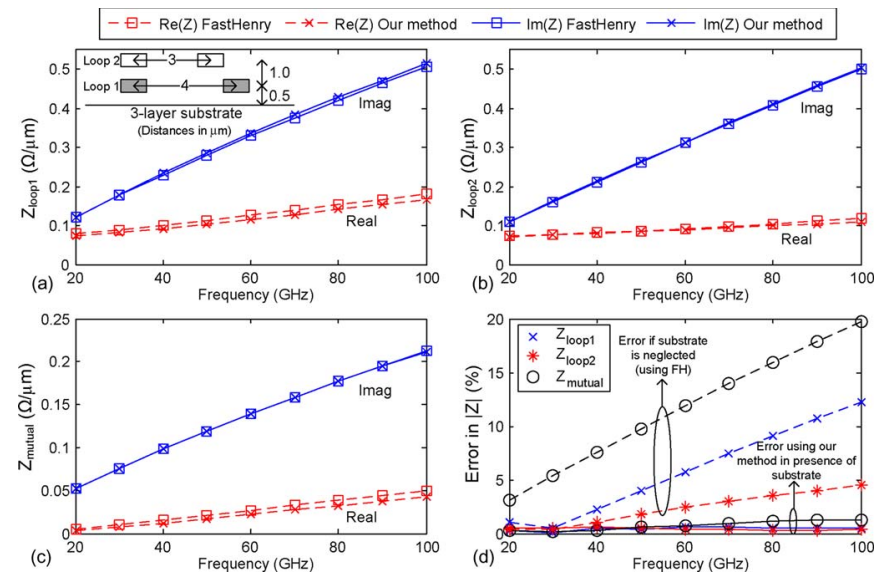

Fig. 14. (a) and (b) Self-impedance and (c) mutual impedance for two conductor loops lying on different metal layers in the presence of a three-layer substrate as a function of frequency. The interconnect configuration is shown in the inset of (a). Each conductor has width $w=1.0 \mu \mathrm{m}$ and thickness $t=0.5 \mu \mathrm{m}$; number of complex images $K=5$ ( $5 \times 3$ filaments used per conductor). (d, Solid lines) Percentage errors in magnitude of impedance calculation using our method with respect to FastHenry for the same configuration. (d, Dashed lines) Error in impedance if the substrate was neglected.

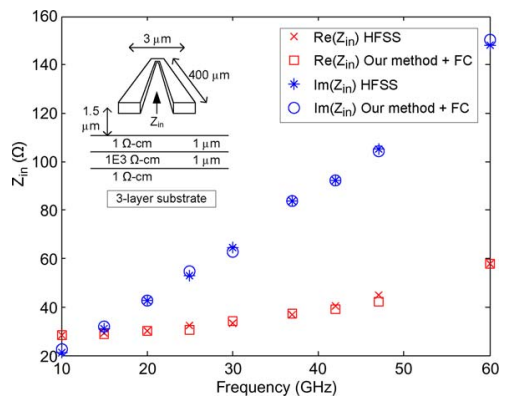

Fig. 15. Impedance $Z_{\text {in }}$ of the one-port network formed by a (inset) closed conductor loop above a three-layer substrate, compared with HFSS [33]. The MQS impedance obtained using our method is combined with capacitance from FastCap [34] using a transmission line model in Spice to obtain the $Z_{\text {in }}$ parameter. Conductor width $1 \mu \mathrm{m}$; thickness $0.5 \mu \mathrm{m}$.

resistance and the inductance per unit length are computed by using our method, and the capacitance is obtained from FastCap [34]. The geometry simulated with HFSS is shown in Fig. 15 (inset). The conductor loop is closed by including additional metal strips at the far and near ends of the loop. The effect of the additional metal is insignificant since the length 


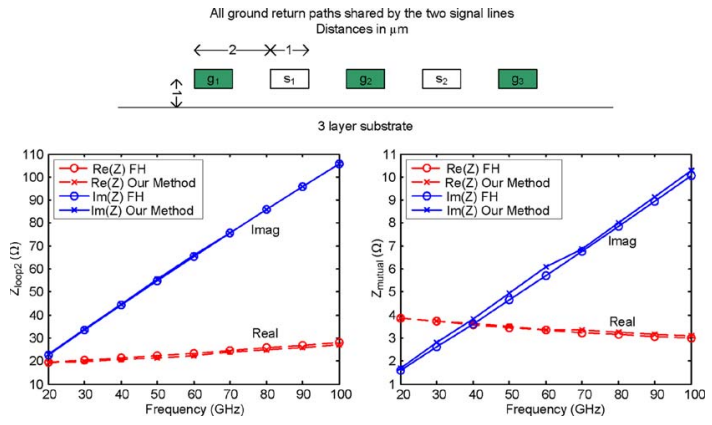

Fig. 16. Self-impedance and mutual impedance of two current loops where the three ground return paths $g_{1}, g_{2}$, and $g_{3}$ are shared by the two signal lines $s_{1}$ and $s_{2}$. Common length of wires $=400 \mu \mathrm{m}$; conductor thickness $=0.5 \mu \mathrm{m}$.
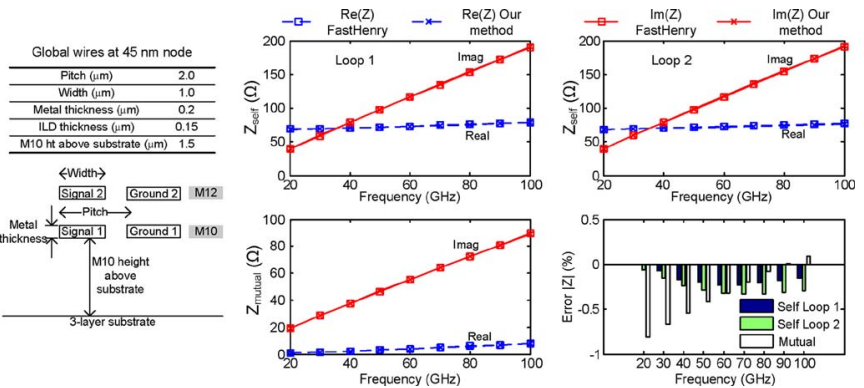

Fig. 17. Self-impedance and mutual impedance for a realistic interconnect geometry at the 45-nm node (shown alongside) and the percent error values with respect to FastHenry. Common length of wires $=400 \mu \mathrm{m}$.

of the conductors is much larger than the transverse separation between them. A small gap $(0.2 \mu \mathrm{m})$ is left at the near end, where a lumped port excites the conductor loop. The results of the comparison are shown in Fig. 15. The maximum error observed in the magnitude of $Z_{\text {in }}$ for this configuration is less than $2 \%$. Note that, for frequencies beyond $60 \mathrm{GHz}$, the results from the HFSS become unstable for the configuration shown.

In the subsequent paragraphs, we compare the accuracy of our impedance extraction methodology with FastHenry for several typical interconnect geometries.

Fig. 16 shows the impedance for a common configuration, where both the signal lines $s_{1}$ and $s_{2}$ share the three ground return paths $g_{1}, g_{2}$, and $g_{3}$ to form two bundles: $s_{1}-g_{1} g_{2} g_{3}$ and $s_{2}-g_{1} g_{2} g_{3}$. Our results for the self-impedance and the mutual impedance show good agreement with FastHenry. Fig. 17 shows the impedance computation results for a realistic global interconnect geometry at the 45-nm node [35]. The interconnect geometry parameters and the configuration are shown alongside. In this case, the error with respect to FastHenry is less than $1 \%$.

Fig. 18 shows a long Manhattan wire with orthogonal segments running on adjacent metal layers M10 and M11 along with a regular grid of ground wires. Each segment of the signal line is routed in close proximity to a segment of the ground grid on the corresponding metal layer-a common design for timing-critical global interconnects to control inductive effects. The mutually perpendicular segments of the Manhattan wire are decoupled (for example, segments $\mathrm{AB}$ and $\mathrm{BC}$ ). On the other hand, the mutual impedance between far-apart parallel segments AB-CD (or between BC and DE) can be neglected because of the large separation $(100-200 \mu \mathrm{m})$ between them.
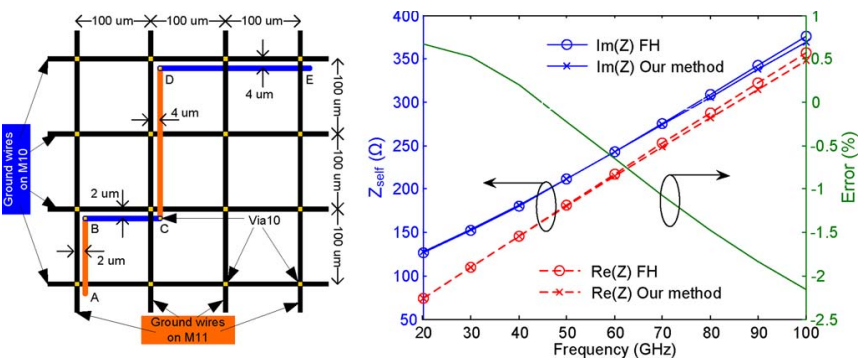

Fig. 18. Impedance of a Manhattan interconnect laid out on metal layers (blue) M10 and (orange) M11 above a three-layer substrate. A grid of ground wires provides nearby return paths to each wire segment. The interconnect geometry parameters are as per global wires at the 45-nm node [35].
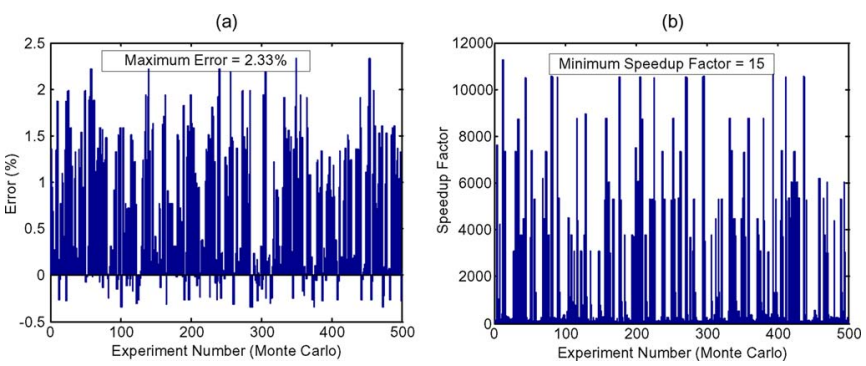

Fig. 19. (a) Error in loop self-impedance computation with respect to FastHenry and (b) speedup in runtime with respect to 2-D PEEC-based computation during Monte Carlo simulation of several randomly generated conductor configurations according to the following parameters: frequency $20-100 \mathrm{GHz}$, pitch 2-8 $\mu \mathrm{m}$, wire width 1-2 $\mu \mathrm{m}$, and height above substrate $1.5-2.2 \mu \mathrm{m}$ (corresponding to metal layers M10 through M12). The interconnect geometry parameters correspond to the global wires at the 45-nm node [35].

Hence, we can separately apply our approach to each linear segment ( $\mathrm{AB}, \mathrm{BC}, \mathrm{CD}$, and $\mathrm{DE})$ to obtain the impedance of the Manhattan wire. As shown in Fig. 18, our results have a maximum error of about $2 \%$ over the entire frequency range of interest.

We run Monte Carlo simulations for a large number of randomly generated interconnect geometries for global wires at the 45-nm node [35] over a wide range of frequencies. The results are shown in Fig. 19(a). The maximum error observed is $2.33 \%$.

\section{B. Computational Efficiency}

Our method for impedance computation is based on the analytical expressions for the substrate Green's function, wherein the substrate boundary conditions are implicit. The only filaments that need to be considered in the solution are those corresponding to the interconnects themselves. On the other hand, both FastHenry and the 2-D PEEC method used for comparison are based on the free-space Green's function. Hence, the substrate layers must be included as explicit conductors. At relevant high frequencies, these layers must be discretized into a large number of filaments, resulting in a large linear system of equations.

Table III compares the computation time with FastHenry to that with our method for impedance extraction. The number of filaments for representing the substrate in FastHenry is chosen by progressively increasing the number of segments in the substrate layers until the result stabilizes. It is observed that the accuracy levels achieved with our method are accompanied 
TABLE III

Computation Cost For SElF-IMPEdance And Mutual-IMPEdance EXTRACTION FOR DIFFERENT INTERCONNECT AND SUBSTRATE Configurations in COMPARISON With FastHenry at $100 \mathrm{GHz} . F_{\text {cond }}$ :

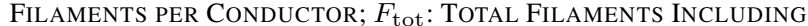
Substrate; $T_{i}$ : Time to Compute the Images (One-Time Cost); $T_{Z}$ : TIME TO COMPUTE THE IMPEDANCE

\begin{tabular}{|c|c|c|c|c|c|}
\hline & & $F_{\text {cond }}$ & $F_{\text {tot }}$ & $T_{i}(\mathrm{~s})$ & $T_{Z}(\mathrm{~s})$ \\
\hline \multirow{2}{*}{ Fig. 10(a) } & FH & 1 & 21640 & - & 517.7 \\
& Our method & 1 & 4 & 91.34 & $<1 \mathrm{sec}$ \\
\hline \multirow{2}{*}{ Fig. 12 } & FH & 15 & 21700 & - & 631.4 \\
& Our method & 15 & 75 & 90.6 & 12.39 \\
\hline \multirow{2}{*}{ Fig. 13 } & FH & 1 & 23360 & - & 99.67 \\
& Our method & 1 & 6 & 90.6 & $<1 \mathrm{sec}$ \\
\hline \multirow{2}{*}{ Fig. 14 } & FH & 15 & 22400 & - & 108.89 \\
& Our method & 15 & 60 & 90.6 & 3.62 \\
\hline
\end{tabular}

TABLE IV

COMPUTATION COST FOR SELF-IMPEDANCE EXTRACTION OF A TWO-CONDUCTOR LOOP OVER A 500- $\mu$ m-WIDE THREE-LAYER Substrate, EACH CONDUCTOR $1 \mu \mathrm{m}$ Wide AND $0.5 \mu \mathrm{m}$ THICK, AT $100 \mathrm{GHz}$. Substrate Profile Is ShOWn In Fig. 6(b). $z_{1}$ : Thickness of The Top Substrate LAyER; $F_{\text {cond }}$ : Number OF FILAMENTS PER CONDUCTOR; $F_{\text {sub }}$ : NUMBER OF FILAMENTS FOR THE SUBSTRATE; $T_{i}$ : Time to Compute the IMAGes (ONE-TIME Cost); $T_{Z}$ : Time to Compute the IMPEDANCE

\begin{tabular}{|c|c|c|c|c|c|}
\hline$z_{1}$ & & $F_{\text {cond }}$ & $F_{\text {sub }}$ & $T_{i}(\mathrm{~s})$ & $T_{Z}(\mathrm{~s})$ \\
\hline \multirow{2}{*}{$1 \mu \mathrm{m}$} & 2D PEEC & 15 & 1323 & - & 110.2 \\
& Our method & 15 & 0 & 90.6 & $<1 \mathrm{sec}$ \\
\hline \multirow{2}{*}{$2 \mu \mathrm{m}$} & 2D PEEC & 15 & 1952 & - & 313.7 \\
& Our method & 15 & 0 & 107.1 & $<1 \mathrm{sec}$ \\
\hline \multirow{2}{*}{$3 \mu \mathrm{m}$} & 2D PEEC & 15 & 2581 & - & 821.5 \\
& Our method & 15 & 0 & 166.9 & $<1 \mathrm{sec}$ \\
\hline \multirow{2}{*}{$4 \mu \mathrm{m}$} & 2D PEEC & 15 & 3829 & - & 2816 \\
& Our method & 15 & 0 & 75.2 & $<1 \mathrm{sec}$ \\
\hline \multirow{2}{*}{$5 \mu \mathrm{m}$} & 2D PEEC & 15 & 4468 & - & 4522 \\
& Our method & 15 & 0 & 146.8 & $<1 \mathrm{sec}$ \\
\hline
\end{tabular}

by almost two orders of magnitude reduction in computation time.

Since FastHenry is applicable to general 3-D interconnect geometries, we also compare our 2-D method with 2-D PEECbased computation in Table IV. The number of filaments in the substrate for the 2-D PEEC must ensure that the filament cross-sections are smaller than the skin depth at the specified frequency. As the thickness of the low-resistivity top layer of the substrate increases to a few micrometers [3], the computation cost with the 2-D PEEC substantially increases due to the larger number of filaments required. On the other hand, with our method, a change in the substrate layer thickness only demands the evaluation of a new set of substrate images while the impedance computation cost remains constant. Hence, Table IV shows orders-of-magnitude improvement in computational efficiency by using our method.

Finally, the speedup observed from Monte Carlo simulations on a large number of randomly generated interconnect geometries is shown in Fig. 19(b). The minimum speedup observed in the self-impedance computation with respect to 2-D PEEC-based computation is 15 .

\section{CONCLUSION}

A general methodology has been presented for selfimpedance and mutual-impedance extraction of VLSI inter- connects in two dimensions in the presence of a multilayered conductive substrate. This approach is based on the Green's function for a magnetic dipole, which naturally leads to current loops giving the correct physical representation for onchip conductor currents. The relevant regimes of distances and frequencies are covered. The resulting equations (21), (22), (26), (30), (32)-(34), and (36), as well as those shown in the Appendix, are simple analytical expressions depending on parameters that can easily be extracted from real exponential least square fits to known formulae. The suitability of this approach for massive extraction problems is self-evident. The proposed method is significantly more accurate than the ACIM, more efficient than the RFFM, and much simpler to realize than the DCIM. In addition, a continuous dipole distribution has been employed to compute the magnetic interaction between conductor loops at very small distances from the source current distribution. This approximation allows us to directly apply the dipole Green's function to self-impedance and mutualimpedance computation. The results show that this approach accurately computes the impedance of VLSI interconnects in cases where the substrate is found to have a significant impact. The approach is applicable to VLSI interconnects, where the conductors have a common length that is much larger than the transverse separations between them. Its suitability for realistic VLSI interconnect configurations subject to this constraint has been shown through a wide range of examples. The saving in computation time as compared with the electromagnetic field solver FastHenry is almost two orders of magnitude.

\section{APPENDIX \\ ANALYTICAL EXPRESSIONS FOR $A_{\mathrm{cont}}^{\mathrm{ver}}$ AND $A_{\mathrm{cont}}^{\mathrm{hor}}$}

In the following equations, the expressions for the free-space terms $A_{\mathrm{src}}^{\mathrm{ver}}$ and $A_{\mathrm{src}}^{\mathrm{hor}}$ and the substrate contribution terms $A_{\mathrm{sub}}^{\mathrm{ver}}$ and $A_{\mathrm{sub}}^{\mathrm{hor}}$ of the magnetic vector potential are separately shown for readability:

$$
\begin{aligned}
A_{\text {cont }}^{\mathrm{ver}}(x, z) & =\int_{0}^{a_{x}} G_{0, \text { dipole }}^{\mathrm{ver}}\left(x, z, x^{\prime}, z^{\prime}\right) \frac{\mu}{2 \pi} I d x^{\prime} \\
& =A_{\mathrm{src}}^{\mathrm{ver}}(x, z)-A_{\mathrm{sub}}^{\mathrm{ver}}(x, z) \\
A_{\mathrm{cont}}^{\mathrm{hor}}(x, z) & =\int_{0}^{a_{z}} G_{0, \text { dipole }}^{\text {hor }}\left(x, z, x^{\prime}, z^{\prime}\right) \frac{\mu}{2 \pi} I d z^{\prime} \\
& =A_{\mathrm{src}}^{\mathrm{hor}}(x, z)+A_{\mathrm{sub}}^{\mathrm{hor}}(x, z) \\
A_{\mathrm{src}}^{\mathrm{ver}}(x, z) & =-\frac{\mu I}{2 \pi} \begin{cases}-\frac{1}{2} \ln \left|\frac{\left(x-a_{x}\right)^{2}+z^{2}}{x^{2}+z^{2}}\right|, & \text { if } \tan \theta=0 \\
\frac{1}{1+\tan ^{2} \theta} \ln \left|\frac{x-a_{x}}{x}\right|, & \text { if } z=x \tan \theta \\
f\left(A, B, C, x, a_{x}\right), & \text { otherwise }\end{cases}
\end{aligned}
$$

where $A=1+\tan ^{2} \theta, B=-2 x-2 z \tan \theta$, and $C=x^{2}+$ $z^{2} . A_{\mathrm{sub}}^{\mathrm{ver}}(x, z)$ is given by

$$
\begin{aligned}
& A_{\mathrm{sub}}^{\mathrm{ver}}(x, z)=-\frac{\mu I}{2 \pi} \\
& \quad \times \begin{cases}\sum_{k} \frac{-\beta_{k}}{2} \ln \left|\frac{\left(x-a_{x}\right)^{2}+\left(z+2 h+\frac{\alpha_{k}}{\gamma_{1}}\right)^{2}}{x^{2}+\left(z+2 h+\frac{\alpha_{k}}{\gamma_{1}}\right)^{2}}\right|, & \text { if } \tan \theta=0 \\
\sum_{k} \beta_{k} f\left(A, B, C, x, a_{x}\right), & \text { otherwise }\end{cases}
\end{aligned}
$$


where

$$
\begin{aligned}
& A=1+\tan ^{2} \theta \\
& B=-2 x+2\left(z+2 h+\frac{\alpha_{k}}{\gamma_{1}}\right) \tan \theta \\
& C=x^{2}+\left(z+2 h+\frac{\alpha_{k}}{\gamma_{1}}\right)^{2}
\end{aligned}
$$

$A_{\mathrm{src}}^{\text {hor }}(x, z)$ is given by

$$
A_{\mathrm{src}}^{\mathrm{hor}}(x, z)=-\frac{\mu I}{2 \pi} \begin{cases}-\frac{1}{2} \ln \left|\frac{x^{2}+\left(z-a_{z}\right)^{2}}{x^{2}+z^{2}}\right|, & \text { if } \tan \phi=0 \\ \frac{1}{1+\tan ^{2} \phi} \ln \left|\frac{z-a_{z}}{z}\right|, & \text { if } x=z \tan \phi \\ f\left(A, B, C, z, a_{z}\right), & \text { otherwise }\end{cases}
$$

where $A=1+\tan ^{2} \phi, B=-2 z-2 x \tan \phi$, and $C=x^{2}+$ $z^{2}$, and $A_{\mathrm{sub}}^{\text {hor }}(x, z)$ is given by

$$
\begin{aligned}
& A_{\mathrm{sub}}^{\mathrm{hor}}(x, z)=-\frac{\mu I}{2 \pi} \\
& \quad \times \begin{cases}\sum_{k} \frac{\beta_{k}}{2} \ln \left|\frac{x^{2}+\left(z+a_{z}+2 h+\frac{\alpha_{k}}{\gamma_{1}}\right)^{2}}{x^{2}+\left(z+2 h+\frac{\alpha_{k}}{\gamma_{1}}\right)^{2}}\right|, & \text { if } \tan \phi=0 \\
\sum_{k} \beta_{k} f\left(A, B, C, z, a_{z}\right), & \text { otherwise }\end{cases}
\end{aligned}
$$

where

$$
\begin{aligned}
& A=1+\tan ^{2} \phi \\
& B=-2 x \tan \phi+2\left(z+2 h+\frac{\alpha_{k}}{\gamma_{1}}\right) \\
& C=x^{2}+\left(z+2 h+\frac{\alpha_{k}}{\gamma_{1}}\right)^{2} .
\end{aligned}
$$

In (39)-(42)

$$
\begin{array}{r}
f\left(A, B, C, x, a_{x}\right) \\
=\frac{2 x+B / A}{\sqrt{-B^{2}+4 A C}}\left[\tan ^{-1}\left(\frac{B+2 A a_{x}}{\sqrt{-B^{2}+4 A C}}\right)\right. \\
\left.-\tan ^{-1}\left(\frac{B}{\sqrt{-B^{2}+4 A C}}\right)\right] \\
\quad-\frac{1}{2 A} \ln \left|\frac{A a_{x}^{2}+B a_{x}+C}{C}\right| \\
f\left(A, B, C, z, a_{z}\right) \\
=\frac{2 z+B / A}{\sqrt{-B^{2}+4 A C}}\left[\tan ^{-1}\left(\frac{B+2 A a_{z}}{\sqrt{-B^{2}+4 A C}}\right)\right. \\
\quad-\frac{1}{2 A} \ln \left|\frac{A a_{z}^{2}+B a_{z}+C}{C}\right| .
\end{array}
$$

\section{REFERENCES}

[1] R. Suaya, R. Escovar, S. Ortiz, K. Banerjee, and N. Srivastava, "Modeling and extraction of nanometer scale interconnects: Challenges and opportunities," in Proc. 23rd Adv. Metallization Conf., 2006, pp. 17-27.

[2] E. Charbon, R. Gharpurey, P. Miliozzi, R. Meyer, and A. SangiovanniVincentelli, Substrate Noise: Analysis and Optimization for IC Design. Boston, MA: Kluwer, 2001.

[3] [Online]. Available: http://www.soitec.com/en/products/

[4] M. Kamon, M. Tsuk, and J. White, "FastHenry: A multipole-accelerated 3-D inductance extraction program," IEEE Trans. Microw. Theory Tech., vol. 42, no. 9, pp. 1750-1758, Sep. 1994
[5] A. Ruehli, "Inductance calculations in a complex integrated circuit environment," IBM J. Res. Develop., vol. 16, no. 5, pp. 470-481, 1972

[6] A. Weisshaar, H. Lan, and A. Luoh, "Accurate closed-form expressions for the frequency-dependent line parameters of on-chip interconnects on lossy silicon substrate," IEEE Trans. Adv. Packag., vol. 25, no. 2, pp. 288-296, May 2002.

[7] H. Ymeri, B. Nauwelaers, K. Maex, S. Vandenberghe, and D. De Roest, "New analytic expressions for mutual inductance and resistance of coupled interconnects on lossy silicon substrate," in Top. Meet. Silicon Monolithic Integr. Circuits RF Syst., 2001, pp. 192-200.

[8] P. Bannister, "Summary of image theory expressions for the quasi-static fields of antennas at or above the earth's surface," Proc. IEEE, vol. 67, no. 7, pp. 1001-1008, Jul. 1979.

[9] V. Okhmatovski and A. Cangellaris, "Evaluation of layered media Green's functions via rational function fitting," IEEE Microw. Wireless Compon. Lett., vol. 14, no. 1, pp. 22-24, Jan. 2004.

[10] R. Escovar, S. Ortiz, and R. Suaya, "An improved long distance treatment for mutual inductance," IEEE Trans. Comput.-Aided Design Integr. Circuits Syst., vol. 24, no. 5, pp. 783-793, May 2005.

[11] A. Deutsch, G. Kopcsay, P. Restle, H. Smith, G. Katopis, W. Becker, P. Coteus, C. Surovic, B. J. Rubin, R. P. Dunne, T. Gallo, K. Jenkins, L. Terman, R. Dennard, G. Sai-Halasz, B. Krauter, and D. Knebel, "When are transmission-line effects important for on-chip interconnections?" IEEE Trans. Microw. Theory Tech., vol. 45, no. 10, pp. 1836-1846, Oct. 1997.

[12] H. Haus and J. Melcher, Electromagnetic Fields and Energy. Cambridge, MA: MIT, 1998. [Online]. Available: http://web.mit.edu/ 6.013 book/www/book.html

[13] K. Coperich, J. Morsey, V. Okhmatovski, A. Cangellaris, and A. Ruehli, "Systematic development of transmission-line models for interconnects with frequency-dependent losses," IEEE Trans. Microw. Theory Tech. vol. 49, no. 10, pp. 1677-1685, Oct. 2001.

[14] A. Niknejad and R. Meyer, "Analysis of eddy-current losses over conductive substrates with applications to monolithic inductors and transformers," IEEE Trans. Microw. Theory Tech., vol. 49, no. 1, pp. 166-176, Jan. 2001.

[15] R. Jiang, W. Fu, and C. C.-P. Chen, "EPEEC: Comprehensive spicecompatible reluctance extraction for high-speed interconnects above lossy multilayer substrates," IEEE Trans. Comput.-Aided Design Integr. Circuits Syst., vol. 24, no. 10, pp. 1562-1571, Oct. 2005.

[16] J. Yang, Y. Chow, and D. Fang, "Discrete complex images of a threedimensional dipole above and within a lossy ground," Proc. Inst. Elect. Eng.-H Microw., Antennas Propag., vol. 138, no. 4, pp. 319-326, Aug. 1991.

[17] G. Dural and M. Aksun, "Closed-form Green's functions for general sources and stratified media," IEEE Trans. Microw. Theory Tech., vol. 43, no. 7, pp. 1545-1552, Jul. 1995.

[18] X. Hu, J. White, J. H. Lee, and L. Daniel, "Analysis of full-wave conductor system impedance over substrate using novel integration techniques," in Proc. 42nd Des. Autom. Conf., 2005, pp. 147-152.

[19] W. Chew, Waves and Fields in Inhomogeneous Media. New York: IEEE Press, 1995.

[20] M. Aksun, "A robust approach for the derivation of closed-form Green's functions," IEEE Trans. Microw. Theory Tech., vol. 44, no. 5, pp. 651-658, May 1996.

[21] M. Yavuz, M. Aksun, and G. Dural, "Critical study of the problems in discrete complex image method," in IEEE Int. Symp. Electromagn. Compat., 2003, pp. 1281-1284.

[22] T. Moselhy, X. Hu, and L. Daniel, "pFFT in FastMaxwell: A fast impedance extraction solver for 3D conductor structures over substrate," in DATE, 2007, pp. 1-6.

[23] J. Jackson, Classical Electrodynamics. New York: Wiley, 1975.

[24] D. Fang, J. Yang, and G. Delisle, "Discrete image theory for horizontal electric dipoles in a multilayered medium," Proc. Inst. Elect. Eng.-H: Microw., Antennas Propag., vol. 135, no. 5, pp. 297-303, Oct. 1988.

[25] Y. Chow, J. Yang, and G. Howard, "Complex images for electrostatic field computation in multilayered media," IEEE Trans. Microw. Theory Tech. vol. 39, no. 3, pp. 1120-1125, Jul. 1991.

[26] F. Ling and J.-M. Jin, "Discrete complex image method for Green's functions of general multilayered media," IEEE Microw. Guided Wave Lett., vol. 10, no. 10, pp. 400-402, Oct. 2000.

[27] M. Yuan, T. Sarkar, and M. Salazar-Palma, "A direct discrete complex image method from the closed-form Green's functions in multilayered media," IEEE Trans. Microw. Theory Tech., vol. 54, no. 3, pp. 1025-1032, Mar. 2006. 
[28] M. Aksun and G. Dural, "Clarification of issues on the closed-form Green's function in stratified media," IEEE Trans. Antennas Propag., vol. 53, no. 11, pp. 3644-3653, Nov. 2005.

[29] G. Golub and V. Pereyra, "The differentiation of pseudoinverses and nonlinear least squares problems whose variables separate," SIAM J. Numer. Anal., vol. 10, no. 2, pp. 413-432, Apr. 1973.

[30] G. Golub and V. Pereyra, "Separable nonlinear least squares: The variable projection method and its applications," Inverse Probl., vol. 19, no. 2, pp. R1-R26, Apr. 2003.

[31] N. Srivastava, R. Suaya, and K. Banerjee, "High-frequency mutual impedance extraction of VLSI interconnects in the presence of a multilayer conducting substrate," in DATE, 2008, pp. 426-431.

[32] F. Grover, Inductance Calculations: Working Formulas and Tables, 2nd ed. New York: Dover, 1962.

[33] User's Guide: High Frequency Structure Simulator (HFSSv10.0), Ansoft Corporation, Pittsburgh, PA, 2005.

[34] K. Nabors and J. White, "Multipole-accelerated capacitance extraction algorithms for 3-D structures with multiple dielectrics," IEEE Trans. Circuits Syst., vol. 39, no. 11, pp. 946-954, Nov. 1992.

[35] Intl. Tech. Roadmap for Semiconductors, 2007. [Online]. Available: http://www.itrs.net/reports.html

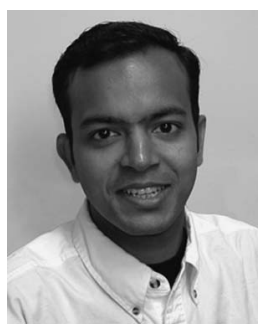

Navin Srivastava (S'03) received the B.Tech. (Honors) degree in electrical engineering from Indian Institute of Technology, Kharagpur, India, in 2000, and the M.S. and Ph.D. degrees in electrical and computer engineering from the University of California, Santa Barbara, in 2006 and 2009, respectively.

From 2000 to 2003, he was with Atrenta Inc., India. $\mathrm{He}$ is currently with the Calibre Division, Mentor Graphics Corporation, Wilsonville, OR. His research interests include very large scale integration interconnects in nanoelectronic circuits.

Dr. Srivastava was the recipient of the Outstanding Graduate Student Paper Award at the VLSI Multilevel Interconnect Conference in 2005 and the IEEE Micro Top Pick Award in 2006.

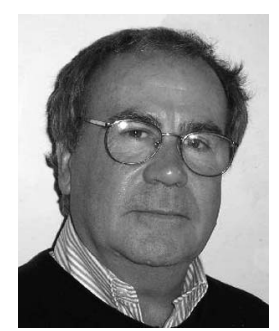

Roberto Suaya (M'99-SM'08) received the Ph.D. degree in physics from the University of Buenos Aires, Buenos Aires, Argentina, with his thesis research done at the Stanford Linear Accelerator Center, Stanford University, Stanford, CA.

He held academic positions in theoretical physics with the University of Illinois, Urbana-Champaign, with McGill University, Montreal, QC, Canada, and with Stanford Linear Accelerator Center, and in computer science with the California Institute of Technology, Pasadena. He held research positions with Fairchild Research Center, with Schlumberger Palo Alto Research in system science, with the Computer Science Laboratory, SRI International (formerly Stanford Research Institute), with Weidlinger Associates, and with the LETI research center of the French Atomic Energy Commission. He is currently the Chief Scientist with the Calibre Division, Mentor Graphics Corporation, St. Ismier, France. He has been an Entrepreneur in CAD, creating Thunder Software. He has extensively published in VLSI CAD and theoretical physics, coedited a book VLSI and Parallel Computation (Morgan Kauffman), and extensively lectured in Europe, North and South America, and Asia.

Dr. Suaya is an Associate Editor of the IEEE TRANSACTIONS ON CAD and a member of the Technical Advisory Board of Semiconductor Research Corporation. He has cochaired international conferences on frontiers in VLSI.

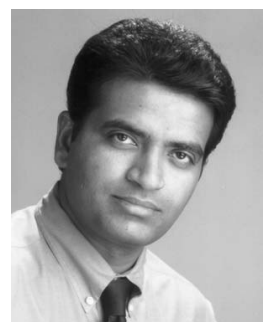

Kaustav Banerjee (S'92-M'99-SM'03) received the $\mathrm{Ph} . \mathrm{D}$. degree in electrical engineering and computer sciences from the University of California, Berkeley, in 1999

From 1993 to 1997, he held summer/visiting positions with Texas Instruments Inc., Dallas, and in 2001, with the Swiss Federal Institute of Technology, Lausanne, Switzerland. From 1999 to 2001, he was a Research Associate with the Center for Integrated Systems, Stanford University, Stanford, CA. From February to August 2002, he was a Visiting Faculty with the Circuit Research Laboratories, Intel, Hillsboro, OR. In July 2002, he joined the faculty of the Department of Electrical and Computer Engineering, University of California, Santa Barbara, where, since 2007, he has been a Ful Professor. His research has been chronicled in over 160 journal and refereed international conference papers and in two book chapters on 3-D ICs. He has also coedited the book Emerging Nanoelectronics: Life With and After CMOS (Springer, 2004). His current research interests focus on nanometer-scale issues in high-performance/low-power VLSI as well as on circuit and system issues in emerging nanoelectronics.

Dr. Banerjee was the recipient of a number of awards in recognition of his work, including a Best Paper Award at the Design Automation Conference (DAC) in 2001, the Association for Computing Machinery (ACM)/Special Interest Group on Design Automation Outstanding New Faculty Award in 2004, an IEEE Micro Top Picks Award in 2006, and an IBM Faculty Award in 2008. He has served on the technical program committees of several leading IEEE and ACM conferences, including the IEEE International Electron Devices Meeting, the Design Automation Conference, the International Conference on ComputerAided Design and the IEEE International Reliability Physics Symposium. From 2005 to 2008, he served as a member of the Nanotechnology Committee of the IEEE Electron Devices Society. He has also served on the organizing committee of the International Symposium Quality Electronic Design at various positions, including the Technical Program Chair in 2002 and the General Chair in 2005. He currently serves as a Distinguished Lecturer of the IEEE Electron Devices Society. 